\title{
Vergütung und Qualität: Ziele, Anreizwirkungen, internationale Erfahrungen und Vorschläge für Deutschland
}

Reinhard Busse, Helene Eckhardt und Max Geraedts

$11.1 \quad$ Einleitung -207

11.2 Ziele von Vergütungssystemen im stationären Sektor - 208

11.3 Vergütungsformen medizinischer Leistungserbringung und deren potenzielle Qualitätseffekte - 209

11.4 Formen der expliziten Qualitätsbeeinflussung durch Vergütungsmodifikationen (P4Q) - 212

11.5 Ein Modell zur Einordnung von P4QVergütungsmodifikationen und grundlegende Überlegungen zu deren Stellenwert - 214

11.6 P4Q in der europäischen Krankenhausversorgung - 216

11.7 Effekte der Qualitätsbeeinflussung durch P4QVergütungsmodifikationen - 219

11.7.1 Wirksamkeit - 219

11.7.2 Kosteneffektivität -221 
11.8 Eine Einordnung von P4Q-Ansätzen in Deutschland und deren bisherigen Nutzung - 222

11.9 Fazit - 226

Literatur - 227 


\section{- Zusammenfassung}

Der Beitrag befasst sich mit der Frage, wie sich das Vergütungssystem zur Qualität der Patientenversorgung verhält bzw. wie diese über das Vergütungssystem befördert werden kann. Vor dem Hintergrund verschiedener Ziele von Vergütungssystemen wird analysiert, wie stark die Anreize in verschiedenen Vergütungsformen zur Qualitätsverbesserung der Versorgung sind. Sodann werden Möglichkeiten der Vergütungsmodifikation mit dem expliziten Ziel der Qualitätssicherung bzw. -verbesserung betrachtet und in ein Modell eingeordnet. Entsprechende „Payfor-quality"-Programme in neun europäischen Ländern werden dargestellt und ein systematischer Review zu deren Effektivität und KostenEffektivität kurz zusammengefasst. Der Beitrag endet mit konkreten Vorschlägen für Deutschland, wie Qualitätstransparenz, die Indikations-, Struktur-, Prozess- bzw. Ergebnisqualität durch Vergütungsmodifikationen auf der Ebene des einzelnen Falls, aller Fälle mit der gleichen Diagnose bzw. allen Fällen eines Krankenhauses verbessert werden kann - und wie weit Deutschland auf diesem Weg bereits ist.

The article deals with the question of how the payment system relates to the quality of patient care and how this can be promoted via the payment system. Against the background of different objectives of reimbursement systems, the authors analyse how strong the incentives of different forms of payment are to improve the quality of care. They consider the possibilities of payment modification with the explicit goal of quality assurance or quality improvement and classify them. They describe pay-for-quality programmes in nine European countries and give a brief systematic review of their (cost)effectiveness. The article concludes with proposals for Germany on how quality transparency, indication, structure, process and outcome quality could be improved through payment modifications at the level of the individual case, all cases with the same diagnosis or all cases of a hospital - and how far Germany is already following this path.

\subsection{Einleitung}

Qualität im Gesundheitswesen findet in den Fachkreisen seit Jahrzehnten eine große Beachtung. Der breiten Öffentlichkeit bekannt wurde die Diskussion um die Qualität in der stationären Versorgung vor allem durch die Analyse der Patientensicherheit in amerikanischen Krankenhäusern (Kohn et al. 2000). Doch auch in deutschen Krankenhäusern ist die Qualität der Versorgung verbesserungswürdig, wie u. a. die von der OECD durchgeführten und veröffentlichten Vergleiche zur Intrahospitalletalität von Patienten mit einem akuten Myokardinfarkt verdeutlichen (OECD 2018). Dabei wird immer wieder auf die potenzielle Steuerungswirkung der Vergütung verwiesen, selbst wenn deren Modifikationen mit dem Ziel der Qualitätsverbesserung hinsichtlich der damit tatsächlich erreichten Effekte wissenschaftlich - und damit auch politisch - umstritten sind.

In Deutschland stammt der vermutlich erste Beitrag zum Zusammenhang von Vergütung und Qualität von 1998, behandelte aber den ambulanten Sektor (Krauth et al. 1998). International begann die Diskussion um „Payfor-Performance" mit den sichtbaren und viel diskutierten Publikationen im Journal of the American Medical Association (Rosenthal et al. 2005), in den Annals of Internal Medicine (Petersen et al. 2006) und Health Affairs (Rosenthal et al. 2007), auch wenn die eigentlichen Studien dazu bereits in den frühen 1990ern begonnen hatten (Busse 2016). Da im globalen Kontext von Gesundheitssystemen der Begriff „Performance“ aber eher im Sinne des „performance assessment" von ganzen Gesundheitssystemen genutzt wird, bei dem es - neben der Qualität der Leistungserbringung im engeren Sinne - auch um die Erreichung von bedarfsgerechtem Zugang (etwa gemessen durch „unmet need") zu bevölkerungsweiten Outcomes (etwa gemessen durch „amenable mortality“) und die Kosten-Effektivität des Gesamtsystems geht, wird in diesem Beitrag konsequent der Begriff „Qualität“ statt Performanz genutzt, etwa als „Pay-for-Quality“ (P4Q). Dieses Kapi- 
tel beginnt mit der Überlegung, welche Ziele mit der Vergütung von Krankenhausleistungen erreicht werden sollen und welche Rolle "Qualität“ dabei spielt ( $\triangleright$ Abschn. 11.2). Es folgt dann ein Überblick über die grundsätzlichen Vergütungsformen medizinischer Leistungserbringung, wobei deren Wirkung auf die eingangs ausgeführten Ziele untersucht wird, insbesondere im Hinblick auf deren potenzielle Qualitätseffekte ( Abschn. 11.3). Anschließend werden die zur expliziten Beeinflussung der Qualität genutzten Vergütungsmodifikationen erläutert ( $\triangleright$ Abschn. 11.4). In $\triangleright$ Abschn. 11.5 wird ein Modell zur Einordnung von P4Q-Vergütungsmodifikationen dargestellt und grundlegende Überlegungen zu deren Stellenwert angestellt.

Es folgt ein Überblick über P4Q-Programme im Krankenhaussektor in europäischen Ländern. Daran anschließend werden empirische Befunde $\mathrm{zu}$ deren Effektivität bzw. Kosten-Effektivität dargestellt. Die - Abschn. 11.6 und 11.7 stützen sich auf den Buchbeitrag Eckhardt et al. (2019), dessen Verwendung für den vorliegenden Beitrag die Autoren zugestimmt haben.

Als Abschn. 11.8 folgt eine umfassende Darstellung der für Deutschland zu diskutierenden qualitätsorientierten Vergütungsmodifikation - einschließlich einer Analyse, welche schon genutzt werden. Ein Fazit beendet das Kapitel als $>$ Abschn. 11.9

\subsection{Ziele von Vergütungssystemen im stationären Sektor}

Jede Diskussion um die potenzielle - und tatsächliche - Beeinflussung der Qualität durch die Vergütung sollte mit der Frage beginnen, welches Spektrum an Zielen eigentlich mit der Vergütung von Krankenhausleistungen verbunden ist. Diese grundlegende Frage wird häufig nicht explizit beantwortet. Die Vergütung von Krankenhäusern soll sicherstellen, dass diese
- Leistungen erbringen und nicht nichts tun,

- sich um die Patienten kümmern, die Bedarf an medizinischen Leistungen haben (d.h. Unterversorgung verhindern) und keine Risikoselektion betreiben (wozu auch gehört, für Notfälle adäquate Reservekapazitäten vorzuhalten),

- nur angemessene - und keine nicht indizierten - Leistungen erbringen (d. h. Fehlund Überversorgung vermeiden),

- die Leistungen in einem personell und technisch angemessen ausgestatteten Setting unter Beachtung die Qualität steigernder Prozesse erbringen, um so hohe Ergebnisqualität zu erreichen und die Patientensicherheit nicht zu gefährden,

- zur Ausgabenkontrolle beitragen,

- die Leistungen effizient erbringen, d.h. Ausgaben und Ergebnis in einem angemessenen Verhältnis stehen und Geld nicht wegen Ineffizienzen verschwendet wird und

- für Patienten, Kostenträger, Allgemeinheit und Politik transparent machen, für welche Patienten(gruppen) welche Leistungen bei welchem Ressourceneinsatz und Ergebnissen erbracht wurden, um eine Steuerung, aber auch kontinuierliche Evaluation und Verbesserung zu ermöglichen.

Diese Aufstellung (ohne den Anspruch auf Vollständigkeit, da z. B. weitergehende Funktionen von Krankenhäusern etwa bei Forschung und Aus- und Weiterbildung fehlen) zeigt erstens, dass die Qualität der Leistungserbringung längst nicht das einzige Ziel ist, das mit der Vergütung von Krankenhausleistungen verbunden ist. Sie zeigt aber auch die Verknüpfung mit übergeordneten Performanzkriterien zur Beurteilung ganzer Gesundheitssysteme; so ist das Ziel, dass sich Krankenhäuser um Patienten kümmern, die Bedarf an medizinischen Leistungen haben, natürlich umso besser zu erreichen, je besser der Krankenversicherungsschutz in einem Land ausgestaltet ist - und die Effizienz der Leistungserbringung in Krankenhäusern wird auch davon abhängen, ob es andere effizientere Versorgungsformen im ambulanten Bereich gibt. 


\subsection{Vergütungsformen medizinischer Leistungserbringung und deren potenzielle Qualitätseffekte}

Bei der Vergütung medizinischer Leistungen reicht das Spektrum der Möglichkeiten von der Vergütung jeder einzelnen erbrachten Leistung bis hin zur Bereitstellung eines globalen Budgets, mit dem alle Leistungen zum Beispiel eines definierten Zeitraums abgegolten werden. Dazwischen finden sich verschiedene Formen der Aggregation einzelner Leistungen, die im Folgenden kurz skizziert werden. Dabei werden deren theoretische, potenzielle Qualitätseffekte holzschnittartig benannt, ohne dass beachtet wird, dass jede der Vergütungsformen mit zusätzlichen, explizit auf die Qualität zielenden Modifikationen verknüpft werden kann. Diese Verknüpfung wird erst im anschließenden Abschnitt behandelt.

\section{- Budget}

Globale Budgets stellen die höchste Aggregationsebene der Vergütung dar. In der Reinform werden den Leistungserbringern Finanzmittel unabhängig von den tatsächlich zu erbringenden Leistungen zur Verfügung gestellt, die zumeist aber auf der Basis der Ausstattung (bei Krankenhäusern also Art und Anzahl an Abteilungen bzw. Betten sowie Personal und technischer Ausstattung), ggf. modifiziert um historische Inanspruchnahmedaten, kalkuliert werden. Da die Budgets typischerweise restriktiv kalkuliert werden, tendieren die Leistungserbringer bei einer solchen Vergütungsform zum einen zur Risikoselektion und zum anderen zur Unterversorgung, sichtbar u. a. an Wartelisten, sodass die Qualität der Versorgung der Population insgesamt leiden kann. Budgets tragen hingegen zu einer guten Ausgabenkontrolle bei.

\section{- Kopfpauschalen}

In die gleiche Richtung gehen auch die Anreizmechanismen bei einer Vergütung auf der
Basis von Kopfpauschalen, die klassischerweise im Bereich der ambulanten Versorgung verwendet werden. Ihre Höhe variiert zumeist mit bestimmten Charakteristika der Patienten, etwa Alter oder dem Vorhandensein von chronischen Erkrankungen, um der Bedarfsorientierung gerecht $\mathrm{zu}$ werden. Das soll die möglichen Nebenwirkungen von Risikoselektion und Unterversorgung dämpfen, wenn pro Kopf der bei einem Leistungserbringer eingeschriebenen Patienten zeitbezogen Finanzmittel zur Verfügung gestellt werden, die unabhängig von seinem Bedarf und den jeweils für die einzelnen Patienten erbrachten Leistungen sind. Gleichzeitig streben die Leistungserbringer nach einer Ausweitung der Anzahl eingeschriebener Patienten, was wiederum die Zeit pro Patient einschränkt und damit negative Auswirkungen auf die Qualität der Versorgung hervorrufen kann.

\section{- Episodenvergütung}

Wird eine gesamte Krankheitsepisode mit allen Leistungen, die zur Diagnostik und Behandlung dieser Episode notwendig waren, pauschal - wiederum auf der Basis historischer Kennwerte - vergütet, dann kann auch diese Vergütungsoption in die Richtung einer Unterversorgung tendieren. Gleichzeitig sind auf der einen Seite Risikoselektionen und auf der anderen Seite das Phänomen des „Upcoding“ - des Höher- bzw. Kränker-Kodierens der einzelnen Fälle, um so die für schwerere Krankheitsepisoden vorgesehenen Vergütungen zu erzielen - zu bedenken. Während Unterversorgung und Risikoselektion sicher mit negativen Effekten auf die Qualität einhergehen, führt das Hochkodieren beim einzelnen Patienten zwar nicht direkt zu einem Qualitätsverlust, jedoch werden Finanzmittel verbraucht, die an anderer Stelle hilfreicher hätten eingesetzt werden können, sodass auch hier theoretisch die Qualität der Versorgung in Mitleidenschaft gezogen werden kann.

\section{- Fallpauschale}

Sehr ähnlich kann auch die Vergütung auf der Basis einer Fallpauschale beurteilt werden. 
Während sie eindeutige Vorteile hinsichtlich der Transparenz (es wird eine unendlich große Anzahl verschiedener Patienten in eine endliche Anzahl an Gruppen eingeordnet) und technischen Effizienz aufweist, besteht die Gefahr von Risikoselektion und Unterversorgung (zumindest von Leistungen, die für die Pauschale nicht konstitutiv sind) - bei gleichzeitiger Tendenz zum Hochkodieren. Hinzu kommt die Gefahr einer Indikationsausweitung hin $\mathrm{zu}$ nicht notwendigen Fällen zur Fallzahlgenerierung und damit Erlössteigerung. Zumindest theoretisch wird bei dieser Vergütungsform erwartet, dass Qualitätssteigerungen auf der Basis einer Spezialisierung und gesteigerter Erfahrung der Leistungserbringer möglich sind.

\section{- Tagespauschalen}

Diese Vergütungsform findet sich typischerweise im stationären Bereich. Um den Erlös zu steigern, wird der Krankenhausaufenthalt um eher unproduktive Tage verlängert, womit u. a. das Risiko für nosokomiale Infektionen steigt. Auch bei dieser Vergütungsform sind Risikoselektionen und eine Unterversorgung (bezogen auf erbrachte Leistungen) zu erwarten. Positive Qualitätsanreize existieren auch bei dieser Vergütungsform nicht.

\section{- Vergütung gebündelter Leistungen bzw. von Leistungspauschalen/-komplexen}

Hierbei werden einzelne Leistungen zusammengefasst, die typischerweise bei der Diagnostik oder Therapie bestimmter Symptome oder Erkrankungen gemeinsam erbracht werden (bspw. die "Ordinationsgebühr" einmal pro Quartal im ambulanten Sektor). Bei dieser Vergütungsform sind Mitnahmeeffekte bei sowieso den Leistungserbringer aufsuchenden Patienten zu erwarten. Eine Erlössteigerung kann durch Wiedereinbestellung zum jeweils nächstmöglichen Abrechnungstermin erzielt werden, womit potenziell eine Überversorgung und damit Patientengefährdung ausgelöst wird; andererseits könnte eine Unterversorgung erfolgen, wenn eine frühere Wiedereinbestellung notwendig gewesen wäre.

\section{- Einzelleistungsvergütung}

Eine echte Einzelleistungsvergütung beruht auf dem Aufwand für die zu erbringende Leistung und erlaubt je nach Land zusätzlich eine gewisse Marge. Damit besteht - insbesondere bei Leistungserbringern, die viel Erfahrung mit dieser Leistung haben - die Gefahr der Überversorgung, und zwar sowohl indem Leistungen bei Patienten ganz ohne Bedarf für die jeweilige Leistung erbracht werden als auch für die unnötige Mehrfacherbringung bei Patienten mit Bedarf. Dadurch besteht eine Tendenz zur Indikationsausweitung mit negativen Effekten für die Qualität der Versorgung. Der potenziell positive Effekt, dass zumindest keine notwendigen Leistungen unterlassen werden, wird durch die potenziell fatalen Folgen einer Überdiagnostik und Übertherapie bei oftmals falsch positiven Testergebnissen mit der Notwendigkeit von Folgeuntersuchungen wettgemacht.

- Tab. 11.1 fasst für die drei wesentlichen im Krankenhaus angewendeten Vergütungssysteme die Anreize hinsichtlich der zu erwartenden Vor-bzw. Nachteile bezüglich der eingangs genannten Ziele zusammen. Dabei kennzeichnet + einen positiven bzw. steigernden Effekt, - einen negativen bzw. senkenden Effekt und 0 eine Situation ohne klare Anreize in die eine oder andere Richtung bzw. dass sowohl positive als auch negative Anreize existieren. Dabei wird deutlich, dass es kein „optimales“ Vergütungssystem gibt - alle haben Vor- und Nachteile. Diese sind hinsichtlich Bedarf, Aktivität und Ausgabenkontrolle insbesondere zwischen Globalbudget und Einzelleistungsvergütung gegensätzlich - und Fallpauschalen zeichnen sich insbesondere durch ihre Effekte auf technische Effizienz und Transparenz aus.

Es wird jedoch auch deutlich, dass keine Vergütungsform rein positive Anreize für gute Qualität bietet. Im Gegenteil: Letztlich besteht bei allen Vergütungsformen die Gefahr, dass deren Anreizmechanismen die Qualität der Versorgung negativ beeinflussen. Daher verwundert es auch nicht, dass so gut wie alle Gesundheitssysteme z.T. aufwändige Instrumente der Qualitäts- und Abrech- 
- Tabelle 11.1 Effekte der wesentlichen drei Vergütungsformen in Krankenhäusern auf Ziele (Erweitert und modifiziert nach Geissler et al. 2011)

\begin{tabular}{|c|c|c|c|c|c|c|c|}
\hline \multirow{3}{*}{$\begin{array}{l}\text { Vergütungs- } \\
\text { form }\end{array}$} & \multicolumn{7}{|c|}{ Vergütungsziele und zu erwartende positive, negative und neutrale Effekte } \\
\hline & \multirow{2}{*}{$\begin{array}{l}\text { Bedarf } \\
\text { (Risiko- } \\
\text { selektion) }\end{array}$} & \multicolumn{2}{|l|}{ Aktivität } & \multirow[t]{2}{*}{ Qualität } & \multirow{2}{*}{$\begin{array}{l}\text { Ausgaben- } \\
\text { kontrolle }\end{array}$} & \multirow{2}{*}{$\begin{array}{l}\text { Technische } \\
\text { Effizienz }\end{array}$} & \multirow[t]{2}{*}{ Transparenz } \\
\hline & & $\begin{array}{l}\text { Zahl der } \\
\text { Fälle }\end{array}$ & $\begin{array}{l}\text { Leistungen } \\
\text { pro Fall }\end{array}$ & & & & \\
\hline Globalbudget & - & - & - & $\begin{array}{l}0 \text { (Gefahr } \\
\text { der Unter- } \\
\text { versorgung) }\end{array}$ & + & 0 & - \\
\hline $\begin{array}{l}\text { Fall- } \\
\text { pauschalen }\end{array}$ & 0 & + & - & 0 & 0 & + & + \\
\hline Einzelleistung & + & + & + & $\begin{array}{l}0 \text { (Gefahr } \\
\text { der Über- } \\
\text { versorgung) }\end{array}$ & - & 0 & 0 \\
\hline
\end{tabular}

nungskontrolle sowie der Förderung der Compliance der Leistungserbringer eingeführt haben, da das soziale Vertrauen in die Leistungserbringer insofern fehlt, als befürchtet wird, dass nicht das Wohlergehen der einzelnen Patientinnen und Patienten und der gesamten Population sowie eine patientenzentrierte Gesundheitsversorgung im Mittelpunkt steht (Mechanic und Schlesinger 1996). Es wird auch verständlich, warum in praktisch allen Ländern diskutiert wird, die qualitätssichernden bzw. -fördernden Aspekte direkt im Vergütungssystem $\mathrm{zu}$ berücksichtigen (vgl. weiter unten). Dabei sollte jedoch bedacht werden, dass die Stoßrichtung solcher Instrumente vom „Haupt“-Vergütungsmechanismus abhängt, d.h. ein auf Einzelleistungsvergütung beruhendes System wird mehr gegen die qualitätsgefährdende Überversorgung tun müssen als ein auf Budgets beruhendes System, wo z. B. Wartelisten eher im Fokus stehen.

Obwohl bei der Beschreibung von Vergütungsformen häufig die Leistungsaggregation innerhalb eines Patienten und über Patienten hinweg betrachtet wird, bietet es sich auch an, die Art von Informationen zu betrachten, die für die Festlegung der Vergütung genutzt werden. Dafür bietet sich ein ursprünglich von Ellis und Miller (2009) entwickeltes Dreieck mit den

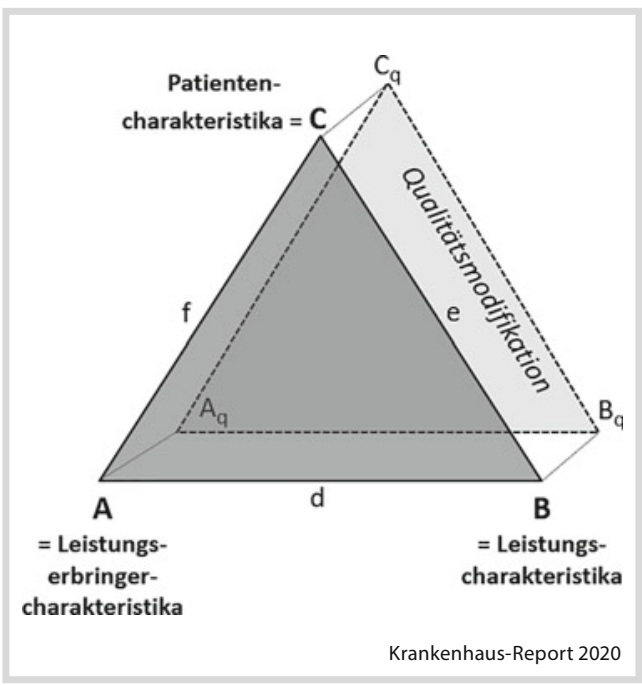

- Abb. 11.1 Informationsgrundlage für wesentliche Vergütungsformen mit prototypischen Ausprägungen $A, B$ und $C$ - und Erweiterung durch Berücksichtigung von Qualitätsmerkmalen $\left(\mathrm{A}_{\mathrm{q}}, \mathrm{B}_{\mathrm{q}}\right.$ und $\mathrm{C}_{\mathrm{q}}$ ) (stark erweitert nach Ellis und Miller 2009)

drei Ecken A, B und C an (vgl. - Abb. 11.1), wobei A für Vergütungsmechanismen steht, bei denen nur Leistungserbringercharakteristika genutzt werden (d. h. etwa Größe und Ausstattung bei Krankenhäusern oder Qualifikation und Erfahrung bei ärztlichen Gehältern), 
B für Leistungscharakteristika (etwa den Zeitaufwand, insbesondere bei Einzelleistungsvergütung) und C für Patientencharakteristika (etwa bei Kopf- und Fallpauschalen, die in ihrer Reinform nur auf Diagnosen und Schweregrad beruhen). In der Realität nutzen viele Systeme Mischformen, etwa indem DRG-Systeme auch die erbrachten Leistungen mitberücksichtigen (und dann in Richtung e tendieren) - oder indem die Einzelleistungsvergütung die Art des Leistungserbringers modifizierend berücksichtigen (und dann eher bei d liegen).

Auch bei dieser Betrachtung wird deutlich, dass bei keiner der drei möglichen Informationsgrundlagen Qualität eine Rolle spielt, sondern diese explizit zusätzlich berücksichtigt werden muss, was durch das nach hinten verschobene Dreieck mit den Ecken $\mathrm{A}_{\mathrm{q}}, \mathrm{B}_{\mathrm{q}}$ und $\mathrm{C}_{\mathrm{q}}$ gekennzeichnet wird. Die Vergütungsmodifikationen, die bisher zur Qualitätssteigerung erprobt wurden, und die Empirie zu deren Effekten werden in den nächsten Abschnitten berichtet.

\subsection{Formen der expliziten Qualitätsbeeinflussung durch Vergütungs- modifikationen (P4Q)}

Die Vergütung von Krankenhausfällen - und die genaue Ausgestaltung der ihr innewohnenden Anreize - sollte andere qualitätsfördernde Maßnahmen unterstützen und nicht konterkarieren. Darin, und weniger als eigenständigem Instrument, liegt die Rolle von „Pay-for-Quality (P4Q)“-Ansätzen.

Dabei werden die beschriebenen Vergütungsinstrumente dahingehend modifiziert, dass die Vergütung mit vorgegebenen Qualitätszielen auf der Ebene der Transparenz, der Indikation, der Struktur, des Prozesses oder des Ergebnisses (s. weiter unten) verknüpft wird und das Erreichen bzw. Nicht-Erreichen von Vorgaben mit positiven oder negativen finanziellen Anreizen verbunden ist.
Das Erreichen von Vorgaben kann dabei im Vergleich, d. h. relativ zu der Leistung der anderen Leistungserbringer (z. B. Belohnung von $20 \%$ der besten Leistungserbringer) oder im Vergleich zu der eigenen historischen Leistung (z. B. Verbesserung um 20 Prozentpunkte im Vergleich zum Vorjahr) gemessen werden. Bei absoluten Qualitätszielen wird das Erreichen einer bestimmten Vorgabe belohnt (z. B. das Erreichen einer 98-prozentigen Impfquote). Verwirrenderweise können auch die Vergütungsmodifikationen selbst - unabhängig von der Definition der Vorgaben - relativ oder absolut sein, d.h. der Bonus könnte z. B. $10 \%$ der vereinbarten Vergütung ausmachen oder pauschal $€ 100$, wodurch er bei DRGs mit niedrigem Relativgewicht eine größere Rolle spielen würde. - Tab. 11.2 fasst die Grundlage der Vergütungsmodifikation und die Art der Vergütungsmodifikation zusammen.

Im Folgenden werden Grundtypen von qualitätsorientierten Vergütungsmodifikationen im Einzelnen dargestellt.

\section{- Bonus}

Bonuszahlungen werden zusätzlich (sog. „neues" Geld) zu der üblichen Vergütung für das Erreichen von Qualitätsvorgaben sowie für eine Verbesserung der Leistungsqualität gezahlt. Es hat sich gezeigt, dass (Bonus-)Zahlungen ab einem bestimmten Niveau höhere Effekte haben: Ogundeji et al. (2016) fanden heraus, dass finanzielle Anreize von mind. $5 \%$ des Jahreseinkommens einen größeren Effekt auf die Leistung der Leistungserbringer haben als kleinere finanzielle Anreize. Ein größerer finanzieller Anreiz reduziert jedoch die Wahrscheinlichkeit der Kosteneffektivität eines Programms. Bonuszahlungen für die Erreichung von im Voraus definierten absoluten Qualitätszielen sind einfach zu handhaben. Für Leistungserbringer bieten sie die Sicherheit, dass sie bei Zielerreichung ausgezahlt werden. Langfristig fehlen jedoch Anreize, sich weiter über das Ziel hinaus zu verbessern, wenn die Leistungsindikatoren nicht regelmäßig angepasst werden (Langdown und Peckham 2014). Dies kann auch dazu führen, dass Jahr für Jahr die bereits vor Jahren 
- Tabelle 11.2 Übersicht zu Grundlagen und Arten der Vergütungsmodifikation

\section{Grundlage für Vergütungsmodifikation}

Status quo im Jahr $t_{1}$

Erreichen eines vorher festgelegten Zieles
Erreichen eines Wertes relativ zu anderen (z. B. Belohnung der besten $20 \%$ der Krankenhäuser)
Art der Vergütungsmodifikation

Absoluter Wert Relativer Wert (z.B.Bonus oder (z.B.Bonus oder Malus von Malus von $Y \%$ $\mathrm{X} \in$ /Fall) auf Relativgewicht)

Krankenhaus-Report 2020

erreichte Leistung aufs Neue belohnt wird, obwohl keine weitere Verbesserung stattgefunden hat. Die Nutzung relativer Verbesserungen für Bonuszahlungen kann zu mehr Effektivität des Programms führen, wenn beispielsweise Verbesserungen auch bei schlechter Ausgangslage belohnt werden, sie kann aber andererseits demotivierend auf diejenigen Leistungserbringer wirken, die schon gut sind und sich wenig verbessern. Zudem gehen Bonuszahlungen auf solcher Grundlage mit einem höheren administrativen Aufwand einher, wodurch die Kosteneffektivität der Programme gemindert werden kann. Insofern sollte zwischen absoluten, relativen und veränderungsbasierten Ansätzen abgewogen und diese eventuell miteinander kombiniert werden.

\section{- Malus/Nicht-Vergütung}

Ein Malus bzw. Vergütungsabschlag verringert den Erlös um eine definierte Geldmenge aufgrund von schlechter Leistung oder nicht erreichten Zielvorgaben. Im Extremfall entspricht der Abschlag der vollständigen Vergütung, was zu einer Nicht-Vergütung führt (z. B. bei extrem schlechter Qualität wie den sog. „never events“ wie etwa Operationsbesteck, das im Körper verbleibt oder die Amputation der falschen Extremität, aber auch z. B. bei von vornherein nicht indizierter Hospitalisierung). Die Nichtvergütung ist für die Kostenträger vermutlich besser zu vermitteln als Abschläge für schlechte Qualität zu bezahlen, was ggf. den Eindruck erwecken könnte, dass sie an der Qualität sparen. Vergütungsabschläge aufgrund von Veränderungen können als diskriminierend be- trachtet werden (eine absolut gesehen gleichhohe Verschlechterung führt bei ungleicher Ausgangshöhe der Qualität zu unterschiedlich hohen Malusbeträgen) und aus diesem Grund zu niedriger Akzeptanz und negativen Verhaltensreaktionen der Leistungserbringer führen. Dennoch können solche Veränderungsmessungen zu einer kontinuierlichen Qualitätsverbesserung führen, wobei die Vergütungsabschläge auf die Leistung aufgrund von Verlustaversion von Individuen einen stärkeren Effekt haben als Bonuszahlungen (Emanuel et al. 2016): Individuen unternehmen eine größere Anstrengung, die erzielten Einnahmen zu sichern, als unsichere Entlohnungen zu verdienen. Somit könnte sich die Verlustaversion der Leistungserbringer positiver auf das Erreichen von Zielen auswirken und damit zu einer positiven Kosteneffektivität beitragen.

\section{- Einbehalte}

Bei den Einbehalten handelt es sich um eine Kombination von Bonus- und Maluszahlungen, wobei ein Betrag am Anfang der Periode einbehalten und entsprechend der Leistung am Ende der Periode umverteilt wird - in diesem Fall werden die Bonuszahlungen aus sog. „alten “ Geldern geleistet. Die Kombination von relativer Messung und Einbehalten (und Umverteilungen) hat ähnliche Vor- und Nachteile wie jene der Bonus- und Maluszahlungen. Jedoch kann die Umverteilung des „alten“ Geldes zusätzlich als ungerecht wahrgenommen werden (Milstein und Schreyögg 2016), was zusätzlich in negativen Verhaltensreaktionen resultieren kann. 


\section{- Shared Savings}

Das primäre Ziel eines Programms mit geteilten Einsparungen ist es, gleichzeitig die Kosten $\mathrm{zu}$ reduzieren und die Versorgungsqualität $\mathrm{zu}$ verbessern, was idealerweise mithilfe einer verbesserten Koordinierung erreicht werden soll (DeCamp et al. 2014). Programme mit geteilten Einsparungen können unterschiedliche Anreizstrukturen haben. Die Teilnehmer (Leistungserbringer) werden dabei an Einsparungen beteiligt, die sich für die Zahler im Vergleich zu einem Referenzwert ergeben, allerdings nur unter der Voraussetzung, dass sie gleichzeitig bestimmte Qualitätsziele erfüllen. Neben den Einsparungen können auch die Risiken zwischen dem Zahler und den Leistungserbringern geteilt werden. Wie bei den anderen Programmen können diese Ziele absolut oder relativ definiert sein (Joynt Maddox et al. 2017). So entstehen Vor- und Nachteile, die bereits bei Bonus, Malus und Einbehalten dargestellt wurden - es werden Fragen nach Gerechtigkeit der Umverteilung der Einsparungen und der Kosten aufgeworfen. Weitere Nachteile sind ein möglicher "trade-off“ zwischen den Zielen, Einsparungen zu generieren oder die Versorgungsqualität zu verbessern, sowie mögliches Konkurrenzdenken in Strukturen, die eigentlich zusammenarbeiten sollten (DeCamp et al. 2014). Es existiert keine klare Evidenz darüber, welche der beschriebenen Anreizstrukturen überlegen ist. In der Theorie können gemischte Vergütungssysteme, welche die unterschiedlichen Anreize miteinander kombinieren, die negativen Auswirkungen von finanziellen Anreizen minimieren. So kann die Kombination von „altem“ und „neuem" Geld sowie von Bonus- und Maluszahlungen in Verbindung mit den relativen Qualitätszielen die Vorteile der finanziellen Anreize ausnutzen, während dadurch einige Nachteile vermieden werden. Die Verlustaversion der Individuen kann ausgenutzt werden, indem am Anfang einer Periode ein Teil der qualitätsbezogenen Geldsumme ausgezahlt und am Ende dieser Periode, gemessen an der erreichten Leistung, angepasst wird. Ein anderer Ansatz besteht darin, die Leistungserbringer zu bestra- fen, wenn die Minimalkriterien nicht erreicht werden, und sie zu belohnen, wenn weitere Qualitätsziele erreicht wurden. Dagegen sollten äußerst wettbewerbsorientierte Strukturen vermieden werden, um die negativen Auswirkungen, die sie mit sich bringen, zu vermeiden. So wird allgemein dazu geraten, mit P4Q-Programmen nicht einzelne Leistungserbringer $\mathrm{zu}$ fokussieren, sondern immer Gruppen von Leistungserbringern gemeinsam unter Risiko zu stellen (Eijkenaar 2013).

\subsection{Ein Modell zur Einordnung von P4Q-Vergütungs- modifikationen und grundlegende Überlegungen zu deren Stellenwert}

Abb. 11.2 präsentiert ein dreidimensionales Modell mit theoretisch $3 \times 4 \times 5=60$ Ansatzmöglichkeiten, für die in Abschn. 11.8 Beispiele genannt werden, die für Deutschland diskutiert werden könnten. Die drei Dimensionen sind dabei (1) die in $>$ Abschn. 11.4 ausgeführten Arten der Vergütungsmodifikationen, (2) die Qualitätsdimension (d. h. Transparenzerstellung über Qualität sowie Indikations, Struktur-, Prozess- und Ergebnisqualität) sowie (3) der Ansatzpunkt der P4Q, d. h. ob beim einzelnen Patienten, bei allen Patienten mit einer bestimmten Diagnose oder DRG oder bei allen Fällen im jeweiligen Krankenhaus. Diese Dimension sollte noch um eine vierte Ebene erweitert werden, nämlich um diejenige aller Krankenhäuser (oder sogar aller Leistungserbringer) in einer Region bzw. für eine definierte Population.

Es ist jedoch nicht genug, sich ausschließlich auf diese dreidimensionale Einordnung von P4Q zu konzentrieren. Stattdessen sollte bei deren Einführung immer auch die gesamte Patientenversorgung in Augenschein genommen werden - eine stückweise Aufmerksamkeit nur auf bestimmte Aspekte der Versorgung kann zu einer Verschlechterung der Versorgungsqualität in Bereichen der Versor- 


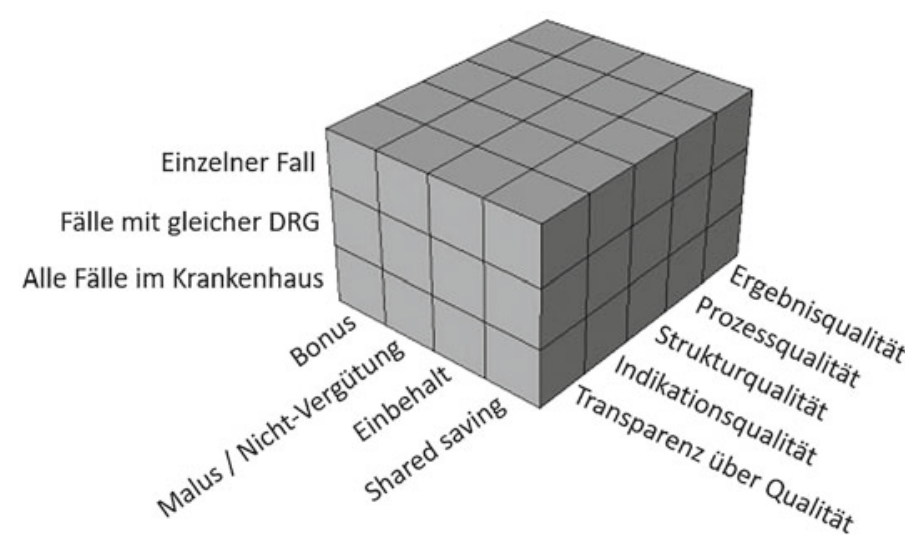

Krankenhaus-Report 2020

- Abb. 11.2 Dreidimensionales Modell zur Einordnung von P4Q-Ansätzen

gung führen, die in der P4Q-Vergütungsmodifikation bzw. im P4Q-Programm nicht adressiert werden. Die Ziele des qualitätsorientierten Vergütungsprogramms sollten im Einklang mit professionellen Normen und Prinzipien stehen - das ist einer der Gründe, warum innovative Versorgungsmodelle gemeinsam mit Leistungserbringern entwickelt und ausgestaltet werden sollten (Doran et al. 2017; Roland und Dudley 2015; van Herck et al. 2010).

Auch die Frage nach der Messung der Qualität ist nicht auf die leichte Schulter zu nehmen. Indikatoren zur Messung der Versorgungsqualität sollten zuverlässig und robust sein. Die Indikatoren können die Strukturen der Leistungserbringer (Charakteristik der Leistungserbringer, Akkreditierungen, Zertifizierungen etc.), Prozesse der Versorgung und die Gesundheitsergebnisse der Patienten widerspiegeln. Während die Prozessindikatoren am häufigsten Anwendung finden - sie sind auch am einfachsten zu messen, sollten jedoch mit Gesundheitsergebnissen korrelieren -, sind die Ergebnisse zwar patientenrelevant, jedoch schwer zu messen. Und noch schwieriger ist es, den $\mathrm{Zu}$ sammenhang zum Programm nachzuweisen. Die Strukturen der Versorgung sollten insbesondere dann angesprochen werden, wenn ein eindeutiger Zusammenhang zwischen der Versorgungsqualität und den Versorgungsstrukturen vorliegt. Um Risikoselektion von Patienten zu vermeiden, sollten Ergebnis-Indikatoren bei der Messung der Qualität risikoadjustiert werden oder aber das Programm sollte gezielt für die Versorgung von Patientengruppen mit vergleichbaren Risiken, etwa dem Vorhandensein einer oder mehreren chronischen Erkrankungen, konzipiert worden sein (Roland und Dudley 2015).

Bei der Implementierung des Programms kann die Akzeptanz dadurch gesteigert werden, dass alle relevanten Interessenvertreter an der Entwicklung, Implementierung und Auswertung des Programms beteiligt werden (Damberg et al. 2014; van Herck et al. 2010). Die teilnehmenden Leistungserbringer sollten ausführlich über Programmlaufzeiten, Anspruchsberechtigungen, Ziele des Programms, die Indikatoren und deren Zusammenhang mit der Versorgungsqualität sowie über die Kriterien für die Auszahlung der Vergütung unterrichtet werden.

Die Programmevaluierung sollte bereits vor der Implementierung des Programms geplant werden (Mehrotra et al. 2009; Damberg et al. 2014; Kondo et al. 2015; Milstein und Schrey- 
ögg 2016). Das Evaluierungsdesign sollte entsprechend dem Ausmaß des Programms (alle vs. ein Teil der Leistungserbringer in einer Region/Land) gewählt werden. Da Studien ohne Kontrollgruppe systematisch den tatsächlichen Interventionseffekt überschätzen (Ogundeji et al. 2016), sollte eine Vergleichskohorte eingeplant werden. Bei fehlender Vergleichsgruppe sollte das Evaluierungskonzept folgende Aspekte berücksichtigen:

- Bereinigung des beobachteten Effekts um den Langzeittrend der natürlichen Qualitätsverbesserung im Zeitverlauf

- Berücksichtigung der Effekte von möglichen konkurrierenden Qualitätsverbesserungsinitiativen

- Ausgangsqualität bei Programmstart

- Überwachung und Evaluierung der Versorgungsqualität außerhalb des Programms

Um verlässliche Ergebnisse zu erhalten, sollten die Daten einige Jahre vor und einige Jahre nach der Implementierung des Programms gesammelt werden. Die Programmevaluierung sollte regelmäßig durchgeführt werden, um positive und negative Programmeffekte zu erfassen und das Programm gegebenenfalls entsprechend anzupassen

\subsection{P4Q in der europäischen Krankenhausversorgung}

In Europa außerhalb von Deutschland konnten insgesamt 13 P4Q-Programme im Krankenhaussektor in neun Ländern identifiziert werden. - Tab. 11.3 bietet eine Übersicht über die identifizierten Programme. Das erste Programm in der Krankenhausversorgung wurde 1998 in Luxemburg eigeführt (FHL 2012), während das neueste Programm 2015 in Kroatien begonnen hat (MSPY 2016). Anfang 2018 hat auch Belgien ein entsprechendes Programm gestartet, sodass jetzt mindestens zehn Länder ein P4Q-Programm aufweisen (FOD Volksgezondheid, Veiligheid van de Voedselketen en Leefmilieu 2018). Typischerweise sind die identifizierten Programme, die mehrheitlich in Krankenhäusern in westeuropäischen Ländern eingeführt wurden, verpflichtender Natur.

Der Fokus der meisten Programme liegt auf der akuten Versorgung von Herz-Kreislauf-Erkrankungen (u. a. bei akutem Myokardinfarkt, Schlaganfall, etc.), Nierenversagen sowie von Hüftfraktur- und Hüft- bzw. Knieersatz-Eingriffen (Programme in England, Frankreich, Italien, Norwegen, Portugal und Schweden). Jedoch werden auch akute Zustände von chronischen Krankheiten wie Diabetes und COPD in einigen Programmen berücksichtigt. Das Ziel der meisten Programme ist, die Wirksamkeit der Behandlung zu verbessern, wobei der Erfolg am häufigsten durch Indikatoren der Prozessqualität (11 der 13 Programme) bzw. der Ergebnisqualität (10 der 13 Programme) gemessen wird. Um dies zu erreichen, werden in Dänemark, England, Frankreich, Kroatien, Luxemburg und Schweden auch strukturelle Vorgaben gemacht. So schreibt das englische „Best Practice Tariff“ der Schlaganfallversorgung vor, dass der Zugang zu der qualifizierten radiologischen und klinischen Auswertung $24 \mathrm{~h}$ am Tag, sieben Tage die Woche verfügbar sein muss, um eine zeitnahe Berichterstattung über die Bildgebung des Gehirns zu ermöglichen (NHS England und NHS Improvement 2019). Üblicherweise werden jedoch die Prozesse des Krankheitsmanagements vorgeschrieben (z. B. die Durchführung eines chirurgischen Eingriffs oder die Einleitung von Behandlung innerhalb einer festgelegten Zeitspanne). In den Programmen in Norwegen (z. B. 5-Jahres-Überlebensraten bei Krebs) und in Kroatien (Gesamtsterblichkeit) werden die finalen Gesundheitsergebnisse gemessen und berücksichtigt.

Patientensicherheit wird in den Programmen in England, Luxemburg, Norwegen und Portugal angesprochen. Das primäre Ziel der Vermeidung von im Krankenhaus erworbenen Infektionen oder von Behandlungsfehlern haben die englischen Programme "Commissioning for Quality and Innovation (CQUIN)“, „Non-Payment for Never-Events“ und „NonPayment for Emergency Readmissions“. 


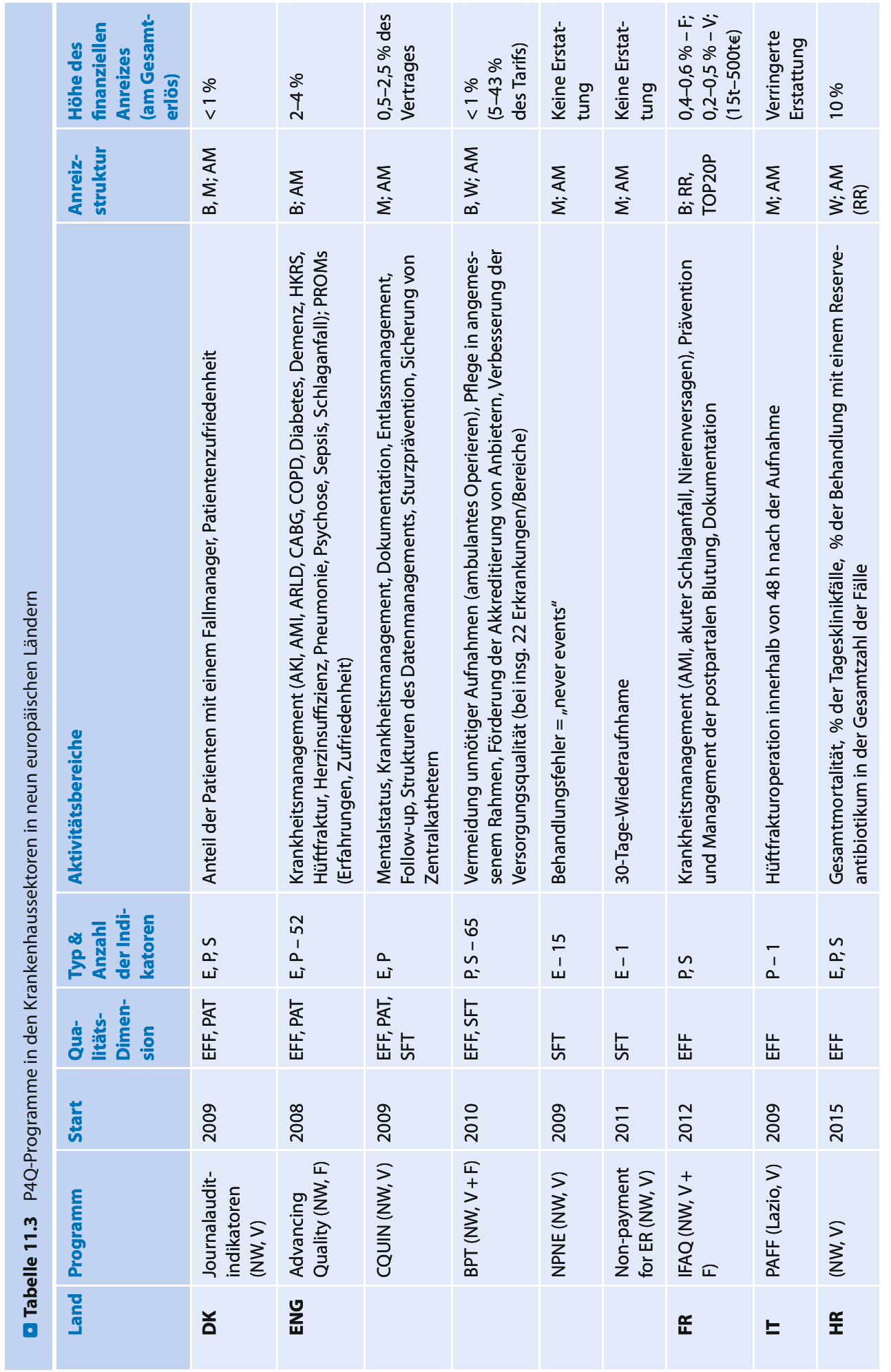




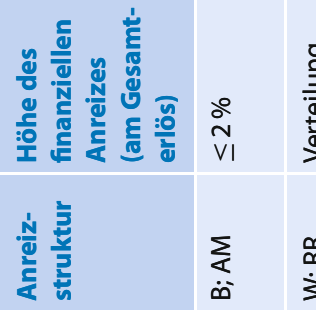

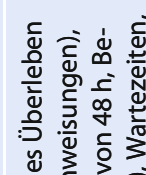

:.ॅ

苍苋产 :

นั

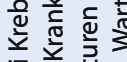

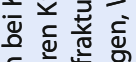

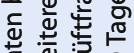

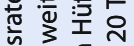

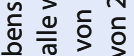

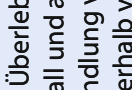

:

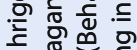

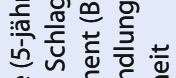

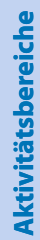

๘

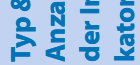

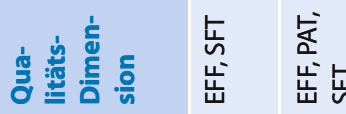

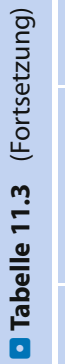

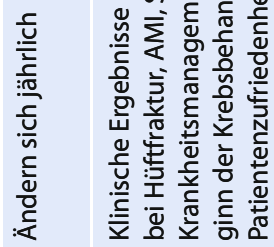

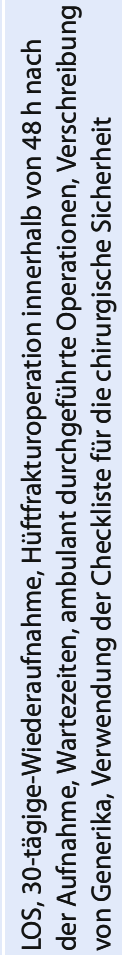
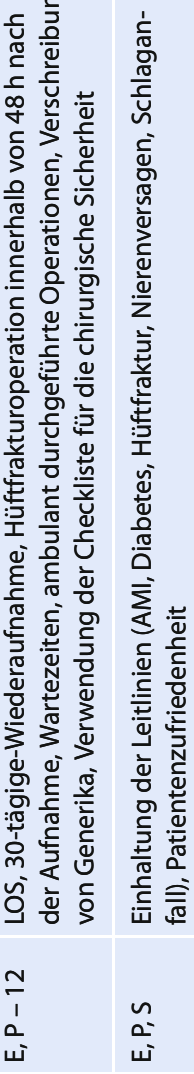

合

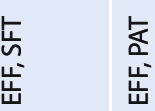

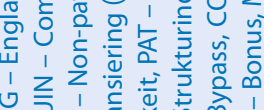

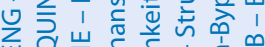
嵌

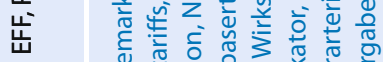

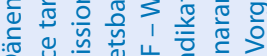

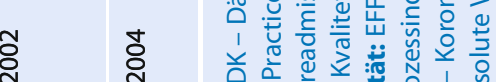

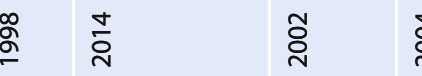

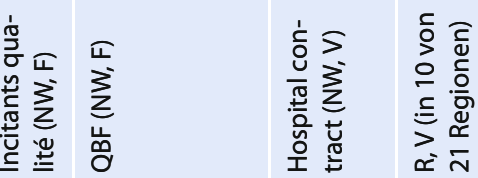

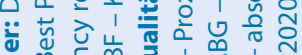
응

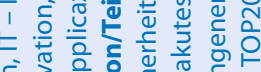

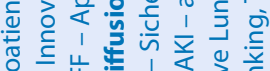

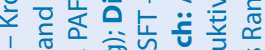

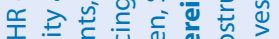

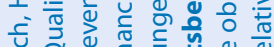

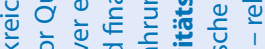

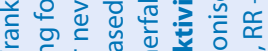

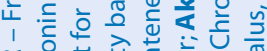
딴 은

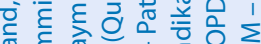
:

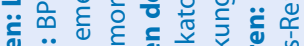
बूँ

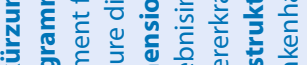

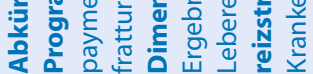


Die Verbesserung von Patientenerfahrungen mit der Krankenhausversorgung wird in den Programmen „Advancing Quality“ und CQUIN sowie in Programmen in Dänemark, Norwegen und Schweden angestrebt (Olsen und Brandborg 2016; AQuA 2017; Anell 2013, S. 43). Dabei werden die gesundheitsbezogene Lebensqualität, Zufriedenheit mit Wartezeiten oder mit Patientensicherheit im Krankenhaus erhoben.

Neun der 13 identifizierten Programme wenden Vergütungsabschläge als finanzielle Anreize an - entweder bleiben die Fälle gänzlich ohne Vergütung, z. B. bei „Non-Payment for Never-Events“, oder es werden Abschläge in Abhängigkeit von der Leistung vorgenommen, z. B. bei CQUIN und bei Programmen in Italien, Norwegen, Portugal und Schweden. Einige der Programme wenden eine Kombination aus Malus- und Bonuszahlungen an. Im dänischen Programm „Journalauditindikatoren“ erhält ein Krankenhaus für die Überschreitung des Schwellenwertes (Mindestleistung) eine Bonuszahlung, während für die Leistungen unterhalb des Schwellenwerts ein Malus anfällt. Die Höhe der Malus- oder Bonuszahlung ist dabei von der Entfernung zum Schwellenwert abhängig (Kristensen et al. 2016). Auch in Portugal und in CQUIN wird eine Kombination angewendet. In Frankreich, Luxemburg und in „Advancing Quality“ werden die Teilnehmer bei Zielerreichung mit einer Bonuszahlung belohnt.

In „Best Practice Tariffs“ erhalten Krankenhäuser, die für bestimmte Leistungsbereiche vom Referenzwert positiv abweichen, eine höhere Vergütung verglichen mit den üblichen DRGs. Die „Best-Practice“-DRGs orientieren sich überwiegend an Parametern für die Prozessqualität, u. a. aus klinischen Leitlinien. Die Vergütung beruht nicht auf den durchschnittlichen Kosten aller Krankenhäuser, sondern auf den Kosten von Krankenhäusern mit guter Prozessqualität und effizienter Leistungserbringung. Im Jahr 2010 wurden vier „BestPractice“-DRGs eingeführt, mittlerweile sind es 20 DRGs bzw. Gruppen von DRGs (NHS Improvement 2019).
Die medizinischen Fachgesellschaften können selbst Vorschläge für "Best-Practice“DRGs einbringen. Die „Best-Practice“-DRGs treffen in England bei Krankenhäusern und medizinischen Fachgesellschaften auf hohe Akzeptanz. Auf robuste Evidenz zu den Wirkungen kann jedoch noch nicht verwiesen werden.

Die Höhe der Bonuszahlungen oder Abschläge variiert zwischen 0,2 und $5 \%$ des jährlichen Krankenhauseinkommens. Lediglich in Kroatien sind $10 \%$ der Erlöse betroffen, die einbehalten und anschließend entsprechend der Leistung ausgezahlt werden. Die Zahlung erfolgt fast immer im Verhältnis zu absoluten Qualitätsvorgaben. Nur in Frankreich (Ministère des Affaires Sociales et de la Santé 2016), Norwegen und Portugal (Srivastava et al. 2016) hängt die Zahlung von der relativen Leistung der Leistungserbringer im Vergleich zu anderen Krankenhäusern ab.

\subsection{Effekte der Qualitäts- beeinflussung durch P4Q- Vergütungsmodifikationen}

\subsubsection{Wirksamkeit}

Eine Übersichtsarbeit über alle bis 2017 veröffentlichten systematischen Reviews ergab insgesamt zwölf Reviews, welche die Effekte von qualitätsorientierten Vergütungsformen im Krankenhaus untersuchten (Armour et al. 2001; Barreto 2015; Christianson et al. 2007, 2008; Mehrotra et al. 2009; Damberg et al. 2007, 2014; Kondo et al. 2015; Korenstein et al. 2016; Milstein und Schreyögg 2016). In jenen Reviews wurden insgesamt 15 unterschiedliche qualitätsorientierte P4Q-Vergütungsformen durch 30 einzelne Studien untersucht. Von diesen analysierten 28 Studien die Effekte von P4Q auf die Prozessqualität, während 13 Studien (auch) Effekte auf die Ergebnisqualität berücksichtigten. Die meisten Studien untersuchten die Effekte von in U.S.-amerikanischen Krankenhäusern imple- 
mentierten Programmen (die im vorherigen Abschnitt nicht enthalten waren).

\section{- Prozessqualität}

Zwei P4Q-Programme wurden ausgiebig untersucht: 17 von 28 Studien untersuchten das von 2003 bis 2009 laufende U.S.-amerikanische Projekt „Premier Hospital Quality Incentive Demonstration“ (HQID), während vier Studien das britische Pendant „Advancing Quality“ (vgl. auch - Tab. 11.3) untersuchten.

Die Reviews stellten auf der Basis einer nur geringen Zahl von qualitativ hochwertigen Studien übereinstimmend fest, dass Effekte auf die Prozessqualität nur von geringem Ausmaß und/oder von kurzer Lebensdauer waren. So waren die Ergebnisse aus der Anfangsphase des HQID zwar hochpositiv, aber nur von kurzer Dauer. In dem heutigen „Hospital Value-based Purchasing Incentive Payment Program (HVBP)“ waren die Effekte positiv, aber statistisch nicht signifikant. In drei weiteren P4Q-Programmen („MassHealth“, „Non-Payment for HACs" des U.S. Centers for Medicare and Medicaid Services - US CMMS, „Baylor Healthcare System“) waren positive Effekte nur bei einzelnen Indikatoren beobachtet worden. Dabei handelt es sich um Indikatoren aus dem Bereich des Akutmanagements des Myokardinfarkts (MI), der Herzinsuffizienz und der außerhalb des Krankenhauses erworbenen Pneumonie. Beispielsweise wurde nur für einen von 19 Indikatoren für die Pneumoniebehandlung (Influenzaimpfung bei Pneumoniepatienten) ein positiver Effekt beobachtet (Damberg et al. 2007; Mehrotra et al. 2009; Damberg et al. 2014; Kondo et al. 2015; Milstein und Schreyögg 2016).

Die Reviews zeigten auch, dass qualitativ weniger hochwertige Studien - d. h. solche ohne eine Vergleichsgruppe - meist positive Effekte auf die Prozessqualität berichteten: Sechs Studien fanden positive Effekte auf Qualitätsindikatoren von Brustkrebs, MI, Herzinsuffizienz, geburtshilflichen Diensten sowie Allgemeinchirurgie. Dagegen fand nur eine VorherNachher-Studie ohne Kontrollgruppe keinen positiven Effekt auf die Behandlungsdauer von
Tuberkulose (Armour et al. 2001; Damberg et al. 2014; Kondo et al. 2015; Mehrotra et al. 2009).

\section{- Ergebnisqualität}

Mit den Effekten auf die Ergebnisqualität verhält es sich ähnlich wie mit der Prozessqualität. Der Rückgang der risikoadjustierten Mortalität assoziiert mit MI, Herzversagen und der außerhalb des Krankenhauses erworbenen Pneumonie war in der Einführungsphase des „Advancing Quality“ größer. Langfristig hatten diese Effekte jedoch keinen Bestand. So kam es, dass 42 Monate nach der Einführung des Programms kein weiterer Rückgang der Mortalität verzeichnet wurde, während Krankenhäuser aus anderen Regionen Englands zur gleichen Zeit sogar einen größeren Rückgang der Mortalität verzeichneten (Damberg et al. 2014; Kondo et al. 2015; Milstein und Schreyögg 2016).

In drei Studien mit vergleichsweise guter Studienqualität konnte zwischen HQID-Krankenhäusern und der Vergleichsgruppe kein Unterschied hinsichtlich der Mortalität assoziiert mit MI, Herzversagen oder Pneumonie festgestellt werden.

Effekte in anderen P4Q-Programmen waren gemischt. Positive Effekte wurden bei unterschiedlichen Gesundheitsergebnissen beobachtet: höherer Anteil an tumorfreien Resektionsrändern, höhere fünf-JahresÜberlebensrate und niedrigere Rezidivraten bei Brustkrebs im taiwanesischen „Breast Cancer Pay for Performance Program (BC-P4P)“ sowie höhere Heilungsraten von Tuberkulose innerhalb von neun Monaten in „Tuberculosis P4P (TB-P4P)“, ebenfalls in Taiwan. Des Weiteren war die Teilnahme an „Blue Cross Blue Shield Michigan P4P (SCBS-P4P)“ assoziiert mit höheren QALY in Bezug auf MI und Herzversagen. Es ist jedoch schwierig, die tatsächlichen Effekte der dargestellten Programme zu bestimmen, da das Studiendesign zum Teil nicht dargestellt wurde (BC-P4P, TB$\mathrm{P} 4 \mathrm{P}$ ) oder aber es fehlen Vergleichsgruppen, die Ergebnisse sind nicht trendbereinigt oder die Effekte können durch konkurrierende Maßnahmen wie "public reporting“ verunreinigt 
worden sein (Kondo et al. 2015; Mehrotra et al. 2009).

\section{- Effekte auf die Patientensicherheit}

Effekte auf die Patientensicherheit wurden in sieben Studien untersucht, die in sechs Reviews eingeschlossen waren. Untersucht wurden Wiederaufnahmeraten, Komplikationen während/nach einem chirurgischen Eingriff sowie im Krankenhaus erworbene Infektionen in sechs Programmen - „BSCS Pilot ACO Program“, „Geisinger ProvenCareSM Integrated Delivery System (Geisinger PCSM)“, „Hawaii Medical Service Association Hospital Pay for Performance Program (HMSAP4P)“, HQID, „MassHealth P4Q Program“ und „Non-Payment for HACs - US CMMS“. Positive und statistisch signifikante Effekte auf vermeidbare Erkrankungen wurden nur durch eine Studie zu „Non-Payment for HACs - US CMMS“ festgestellt (Damberg et al. 2014; Korenstein et al. 2016), während in sechs Studien zu BSCS ACO, Geisinger PCSM, HMSAP4P, HQID und MassHealth keine Effekte auf Wiederaufnahmeraten, Komplikationen während/nach einem chirurgischen Eingriff sowie im Krankenhaus erworbene Infektionen festgestellt werden konnten (Damberg et al. 2014; Kondo et al. 2015; Christianson et al. 2008; Korenstein et al. 2016; Mehrotra et al. 2009; Milstein und Schreyögg 2016).

\section{- Erfahrungen der Patienten}

Erfahrungen der Patientinnen und Patienten mit der Gesundheitsversorgung wurden in vier Studien und fünf Reviews untersucht. Reviews von Kondo et al. (2015) und Milstein und Schreyögg (2016) haben keine Evidenz für verbesserte Patientenerfahrungen mit der Gesundheitsversorgung nach der Einführung von HVBP gefunden. Sie fanden sogar einen leicht negativen Einfluss des Programms auf die Patientenerfahrungen, der allerdings statistisch nicht-signifikant war. Die Patientenzufriedenheit mit der Krankenhausversorgung im HMSA-P4P hat sich um nur wenige Prozentpunkte verbessert, darüber hinaus wurde die statistische Signifikanz nicht berechnet und die Studie enthielt keine Vergleichsgruppe, sodass auch dieses Ergebnis nicht zuverlässig ist (Christianson et al. 2008; Damberg et al. 2014; Mehrotra et al. 2009).

\subsubsection{Kosteneffektivität}

Die Kosteneffektivität wurde lediglich durch ein Review untersucht - Emmert et al. (2012) fanden drei vollständige und sechs partielle ökonomische Bewertungen. Weitere Reviews von Christianson et al. (2007, 2008), van Herck et al. (2010), Hamilton et al. (2013), Damberg et al. (2014) und Kondo et al. (2015) fanden zusätzliche partielle ökonomische Bewertungen, jedoch betrafen die meisten der identifizierten ökonomischen Bewertungen die Kosten und Programmeffekte im ambulanten Sektor.

Allgemein betrachtet haben durch Reviews identifizierte ökonomische Bewertungen eine Reihe an Schwächen: In den meisten Auswertungen wurden die Prozessqualität auf der Effektseite und die Kosten der Zahler auf der Kostenseite berücksichtigt. Administrative Kosten der Leistungserbringer wurden selten berücksichtigt. Darüber hinaus wurden die berücksichtigten Kosten nicht im Detail beschrieben (Emmert et al. 2012). Auch die Designs der eingeschlossenen Analysen weisen eine Vielzahl an Schwächen auf, welche die Zuverlässigkeit der Schlussfolgerungen hinsichtlich der Kosteneffektivität einschränken (Emmert et al. 2012; Mehrotra et al. 2009). Zum Beispiel fehlt eine Bereinigung der Effekte konkurrierender Qualitätsverbesserungsinitiativen, sodass der Effekt nicht eindeutig auf das P4Q-Programm zurückgeführt werden kann (Christianson et al. 2007; van Herck et al. 2010; Kondo et al. 2015). In den meisten Studien sind die Stichprobengrößen sehr klein (Houle et al. 2012; Damberg et al. 2014; Kondo et al. 2015) und in mindestens einer Studie weicht die Analyseeinheit von der Allokationseinheit der Studie ab (Giuffrida et al. 2000).

Des Weiteren kann von Kostenwirksamkeit nicht gesprochen werden, wenn die Wirksamkeit der Programme nicht nachgewiesen wer- 
den kann. Einige Programme im Krankenhaussektor, wie HQID und HVBP, zeigten jedoch nur in den ersten Jahren nach der Einführung des Programms eindeutig positive Ergebnisse hinsichtlich der Wirksamkeit. Dementsprechend kann es auch nur in dieser Zeit Kosteneffektivität gegeben haben und nur dann, wenn die positiven Effekte die (administrativen und die Vergütungs-)Kosten überwiegen. Die Mehrheit der untersuchten Programme wiesen keine positiven Effekte auf die Programmergebnisse auf: Da sie mit Sicherheit zusätzliche Kosten verursachten, können jene Programme nicht kosteneffektiv sein. Diese Schlussfolgerungen gelten für das Krankenhaus und den ambulanten Sektor gleichermaßen.

\subsection{Eine Einordnung von P4Q- Ansätzen in Deutschland - und deren bisherigen Nutzung}

In den vorhergehenden Abschnitten konnte gezeigt werden, dass es aktuell relativ wenige Studien zu den Effekten von P4Q gibt, obwohl eine Vielzahl an P4Q-Programmen im stationären Bereich existieren (Eckhardt et al. 2019). Viele dieser Programme sind breit angelegt und zielen auf die Prozess- und Ergebnisqualität, um die Vergütung des gesamten Krankenhauses zu beeinflussen. In Deutschland werden zum Teil nur solche Programme, bei denen die Prozess- bzw. Ergebnisqualität retrospektiv gemessen wird und daraus - normalerweise mit zeitlicher Verzögerung - finanzielle Konsequenzen für das Krankenhaus folgen, als „Payfor-Performance" (bzw. in unserer Terminologie Pay-for-Quality) subsummiert. ${ }^{1}$

Dabei wird übersehen, dass es jenseits solcher Programme sehr viele andere Möglichkei-

Vgl. etwa „Pay-for-Performance als spezielle strategische Vergütungsform meint in diesem Zusammenhang die retrospektive Koppelung der Vergütung an das von einem Versorger erbrachte, durch Kennzahlen nachgewiesene Leistungsniveau" (Hertle und Veit 2012). ten gibt, die Vergütung im Sinne einer Qualitätssicherung und -verbesserung zu modifizieren. In - Tab. 11.4 werden eine Anzahl solcher Möglichkeiten aufgezeigt, und zwar anhand von zwei Dimensionen aus - Abb. 11.2, nämlich der Qualitätsdimension und dem Ansatzpunkt, woraus sich eine Strukturierung in $5 \times 3=15$ Felder ergibt, angefangen von Vergütungsmodifikationen beim einzelnen Patienten für das (Nicht-)Herstellen von Qualitätstransparenz oben links bis zu Vergütungsmodifikationen bei allen Patienten eines Krankenhauses für das (Nicht-)Erreichen bestimmter Ergebnisse im Sinne der „Pay-for-Quality“Programme etwa in den USA oder Großbritannien unten rechts.

Farblich hinterlegt ist jeweils der Grad der bisherigen Umsetzung in Deutschland, wobei dunkelblau für rechtlich und de facto existent steht, hellblau für einen mittleren Grad der Umsetzung (d. h. rechtlich möglich, aber noch gar nicht oder vereinzelt eingesetzt wie bei den Qualitätsverträgen bzw. eingeschränkt genutzt wie bei der Fallzusammenführung bei ungeplanten Wiederaufnahmen) und grau für rechtlich noch nicht möglich, was bei der Mehrheit der Optionen der Fall ist.

Nur drei Einträge sind dunkelblau markiert - und zumindest zwei davon werden in der deutschen Diskussion nicht unbedingt mit P4Q in Verbindung gebracht, nämlich der Vergütungsabschlag bei unvollständiger Qualitätsdokumentation und die Fehlbelegungsprüfungen durch den MDK.

\section{- Vergütungsabschlag für unvollständige Qualitätsdokumentation}

Dieser Vergütungsabschlag bestand zwar bereits seit längerem, war aber bisher kaum wirksam, da er erst beim Unterschreiten einer Dokumentationsquote von $80 \%$ griff. Die Wahrscheinlichkeit von Sanktionen ist durch das Hochsetzen der Grenze auf $100 \%$ ab dem Erfassungsjahr 2018 deutlich größer geworden. Auch die Wirksamkeit der Abschlagszahlung wegen fehlender Datensätze dürfte sich erhöht haben, seit die Abschlagszahlung wegen auch im Vorjahr fehlender Daten von $€ 150$ auf $€ 300$ 

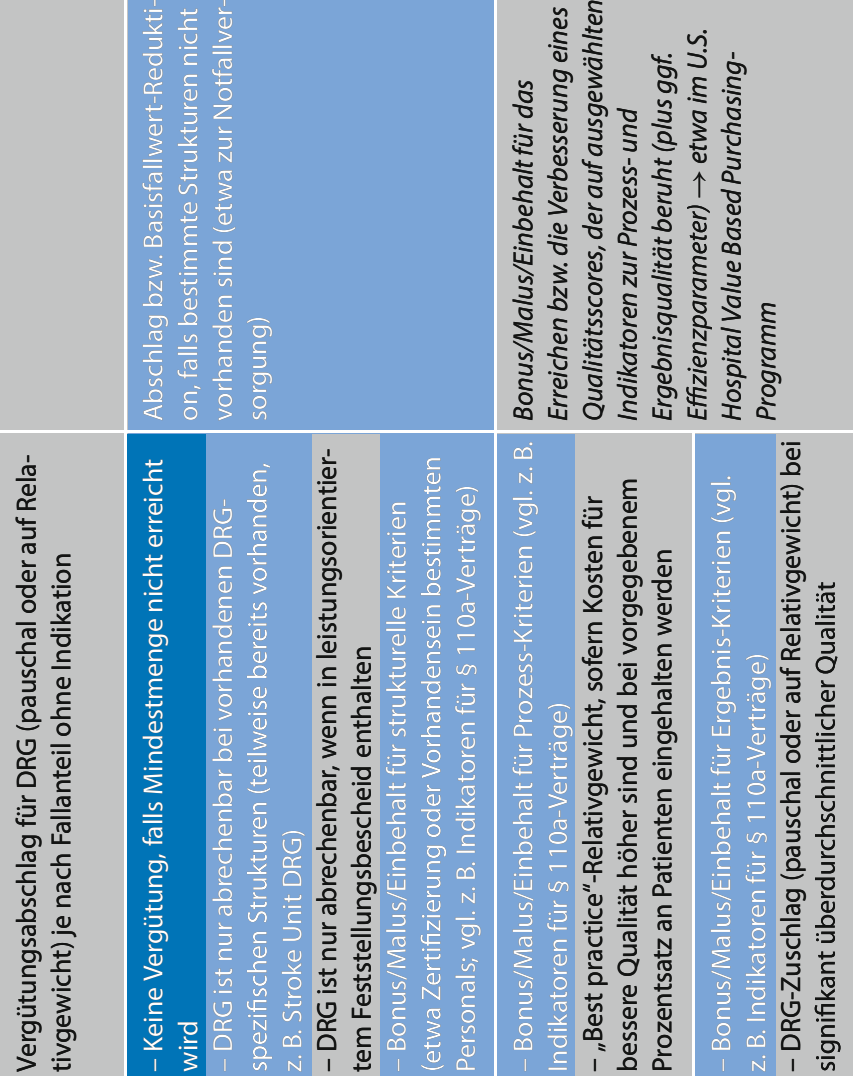

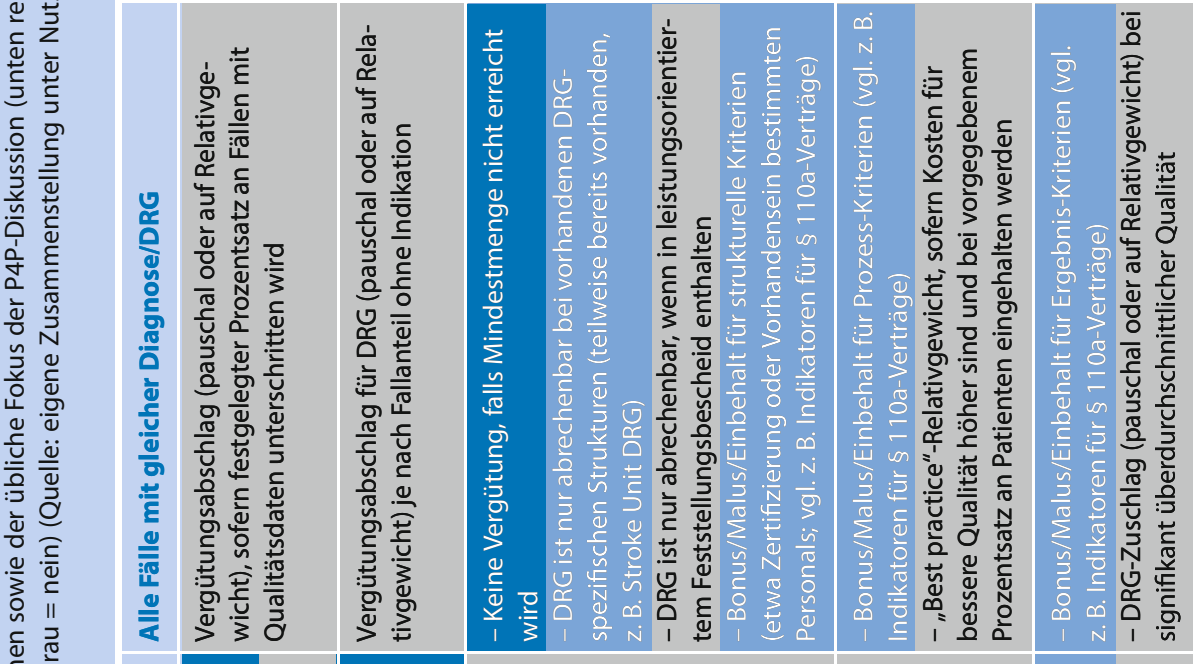

c

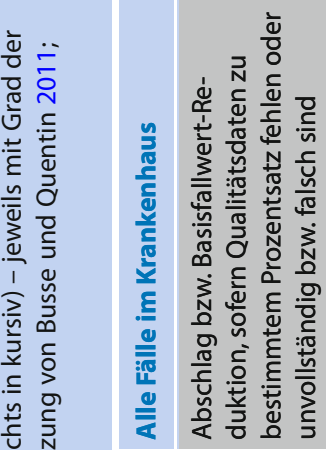

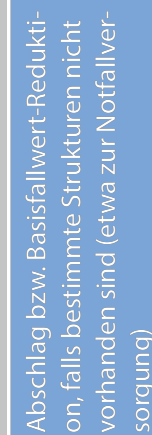

气

$\tilde{g} \stackrel{\circ}{5}$

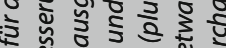

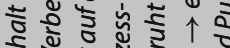

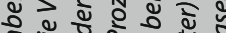

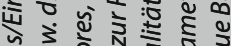

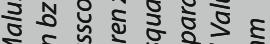

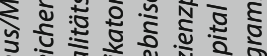

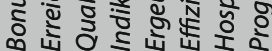

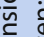

है

总安

: :

픈

○

는

는

पूँ

这

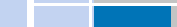

$1+\frac{1}{2}$

(

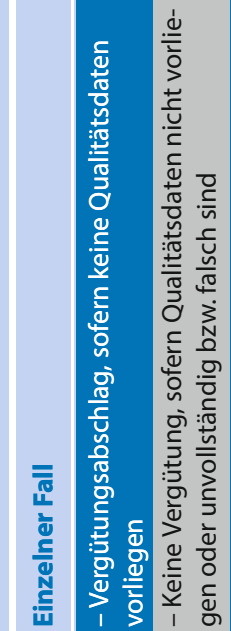

들

竞 춘

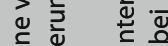

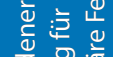

잉

든 $\frac{0}{\frac{C}{2}}$

文选离

든

व

宊

일.

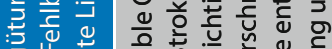

원

> :

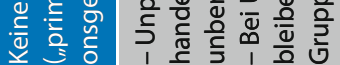

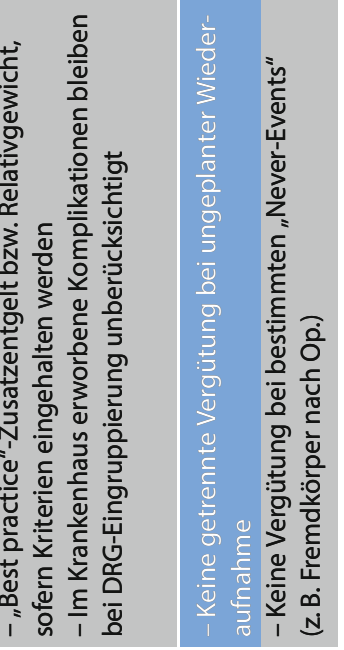

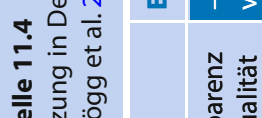

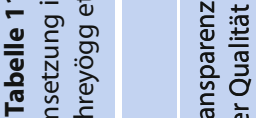

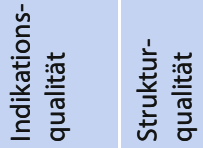

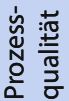

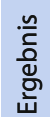

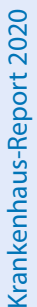


ab dem Erfassungsjahr 2015 verdoppelt wurde. Es scheint jedoch keine öffentlich zugänglichen Daten zu geben, aus denen hervorgeht, wie viele Fälle mit einem Vergütungsabschlag belegt worden sind. In Deutschland betrifft der Vergütungsabschlag nur die nicht dokumentierten Fälle; denkbar wären aber auch Abschläge für alle Patienten mit der entsprechenden Indikation oder sogar für alle Fälle des jeweiligen Krankenhauses.

\section{- MDK-Prüfungen auf nicht indizierte stationäre Aufenthalte}

Die MDK-Prüfungen mit der möglichen Konsequenz der Vergütungsminderung (bis hin zur vollständigen Nicht-Vergütung) werden fast nie unter dem Aspekt der Qualitätssicherung diskutiert (es dominiert das Thema der Fehlabrechnungen), obwohl es etwa beim MDK Nordrhein 2018 in $14 \%$ der Überprüfungen um den Verdacht auf primäre Fehlbelegung (d.h. die nicht indizierte stationäre Behandlung) ging - und in weiteren $43 \%$ um sekundäre Fehlbelegung (MDK Nordrhein 2018), was durchaus durch die mit Krankenhausaufenthalten verbundenen Risiken auch Qualitätsaspekte beinhaltet.

\section{- Keine Vergütung für Leistungen unterhalb der Mindestmenge}

Das Behandlungsergebnis kann in besonderem Maße von der Häufigkeit und der Regelmäßigkeit abhängig sein, mit der ein Arzt oder ein Krankenhaus Leistungen erbringt, die ein hohes Maß an Routine erfordern. Aus diesem Grund wurde 2002 die Mindestmengenregelung bei gut planbaren Prozeduren eingeführt, ohne dass dies jedoch mit klaren finanziellen Implikationen untermauert wurde.

Dies dürfte auch erklären, warum die Mindestmengenregelung weitgehend unterlaufen wurde: So hat eine Analyse für 2006 bis 2010 gezeigt, dass keine Konzentration von Behandlungsfällen auf Zentren mit hohen Fallzahlen stattgefunden und die Anzahl der Krankenhäuser mit sehr geringen Fallmengen sich über die Jahre nicht verändert hat (Cruppé et al. 2015). Auch in den nachfolgenden Jahren scheinen die
Fallzahlen in den Krankenhäusern mit Fallzahlen unterhalb der Mindestmenge nicht gesunken zu sein (Nimptsch et al. 2017). Der Anteil der Fälle von Leber- und Nieren-TX sowie von Knie-TEP in Krankenhäusern unterhalb der Mindestmenge scheint sogar zu steigen. Diese Tendenzen können insbesondere darauf zurückgeführt werden, dass die Krankenhäuser bei Unterschreitung der Mindestmenge eine Vielzahl von Ausnahmeregelungen in Anspruch nehmen können. So wird die Leistung vergütet, wenn das Krankenhaus die Leistung aus dem Katalog planbarer Leistungen erstmals oder nach einer mindestens 24-monatigen Unterbrechung erneut erbringt. Eine Ausnahme gilt auch für eine Leistung, die im Notfall erbracht wurde oder wenn eine Verlegung der Patientin in ein Krankenhaus, das die Mindestmenge erfüllt, medizinisch nicht vertretbar war (vgl. G-BA 2019; $\$ 4$ Abs. 4 S. 3 Mm-R G-BA).

Dies hat sich durch das Krankenhausstrukturgesetz geändert: Zur Erbringung der planbaren Leistungen mit einer verbindlich festgelegten Mindestmenge sind nur noch jene Leistungserbringer berechtigt, die die maßgebliche Mindestmenge je Arzt oder Standort eines Krankenhauses im vorausgegangenen Kalenderjahr erreicht haben (vgl. G-BA 2019; $\$ 4$ Abs. 1 S. 2 der Mm-R G-BA). Auch werden die Leistungen, die unterhalb der Mindestmenge erbracht werden, seit 2018 von der gesetzlichen Krankenversicherung nicht mehr vergütet. Allerdings wurden die Ausweichstrategien durch die Angabe eines Ausnahmetatbestandes nicht geschlossen, sodass abgewartet werden muss, ob die Neuregelung tatsächlich dazu führt, dass Leistungen unterhalb der Mindestmenge nicht mehr vergütet - und damit zukünftig auch tatsächlich nicht mehr erbracht werden (de Cruppé und Geraedts 2018).

\section{- - Steuerung durch strukturelle Vorgaben}

Eine einfache qualitätsorientierte Grundregel wird - außer seit kurzem bei den Mindestmengen - in Deutschland bisher nicht angewendet: Ein Krankenhaus, das personell oder technisch nicht adäquat ausgestattet ist, um einen bestimmten Fall zu behandeln, erhält dafür 
- außer im Notfall bis zur Verlegung - keine Vergütung. Derzeit gelten nur indirekte finanzielle Anreize, indem etwa die Kodierung der OPS 8-981, die normalerweise mit der Behandlung in einer Stroke Unit verbunden ist, zu einer Einstufung in eine andere, höher dotierte DRG führt. Obwohl eine Behandlung in Stroke Units nachweisbare Vorteile für den Patienten hat, dürfen derzeit auch Krankenhäuser ohne eine solche Einheit Patienten mit Schlaganfall behandeln und dafür vergütet werden - genauso wie Krankenhäuser ohne Koronarangiographieeinheit Patienten mit akutem Myokardinfarkt behandeln dürfen und dafür die volle Vergütung erhalten. Hier müsste sowohl die Krankenhausplanung verändert werden, indem der Feststellungsbescheid nicht auf Fachabteilungen und Betten, sondern auf konkrete Leistungsbereiche bzw. -gruppen abzielt, für die jeweils die notwendigen personellen und technischen Voraussetzungen nachzuweisen sind (wie jetzt in Nordrhein-Westfalen vorgesehen), als auch die Krankenhausvergütung umgestellt werden.

Ein Bereich, in dem sich ggf. ein Umdenken in diese Richtung abzeichnet, ist die Notfallversorgung. So hat der G-BA am 19.04.2018 die Strukturen der Notfallversorgung und die strukturellen Vorgaben an die Krankenhäuser neu geregelt (G-BA 2018). Das Ziel dieser Regelung ist, die Qualität der Notfallversorgung durch strukturelle Vorgaben zu verbessern und möglichst die Konzentration von anspruchsvollen Fällen in der Notfallversorgung zu fördern. Die Regelung macht Vorgaben zu der apparativen und personellen Ausstattung von Notfallaufnahmestellen der jeweiligen Stufe der allgemeinen und speziellen Notfallversorgung. An die Einrichtungen mit spezieller Notfallversorgung werden besondere apparative und strukturelle Mindestanforderungen für qualitativ angemessene Versorgung gestellt (GBA 2018). Auch die Vergütung der Notfallversorgung wurde neu geregelt, allerdings nur additiv durch zusätzliche gestufte Pauschalen zur Abdeckung von Vorhaltekosten. Dagegen wurde die Sanktionierung der Nichtteilnahme an der Notfallversorgung durch einen Abschlag pro vollstationären Behandlungsfall prinzipiell beibehalten - es kann nur besser kontrolliert werden. Konsequent wäre es, wenn diese Krankenhäuser das Recht auf die Abrechnung von Notfällen ganz verlieren würden.

Diese Veränderungen wurden in die Wege geleitet, da viele Krankenhäuser die minimalen Strukturanforderungen nicht erfüllt haben. Im Jahr 2017 verfügten 80,8 \% der befragten Krankenhäuser $(n=673)$ rund um die Uhr (24/7) über ein Computertomographiegerät, nur 43,7\% der befragten Krankenhäuser verfügten über die Möglichkeit der perkutanen koronaren Intervention (PCI) 60 min nach Krankenhausaufnahme ggf. in Kooperation und nur $25,1 \%$ konnten eine PCI 20 min nach Krankenhausaufnahme durchführen (IGES 2018). Diese Ergebnisse deuten darauf hin, dass nicht alle Krankenhäuser, die aktuell an der Notfallversorgung teilnehmen, dies auch in Zukunft werden tun können. Voraussichtlich wird nur ein Teil dieser Krankenhäuser alle Bedingungen erfüllen können.

\section{- Qualitätsverträge}

Das übergeordnete Ziel der Qualitätsverträge nach dem durch das KHSG eingeführten $\$ 110 \mathrm{a}$ SGB V ist die Erprobung von finanziellen (und nicht-finanziellen) Anreizen und höheren Qualitätsanforderungen an Strukturen, Prozesse und/oder Ergebnisse der Versorgung im stationären Bereich (•Tab. 11.4 hellblaue Markierung). Im Mai 2017 hat der G-BA gemäß $\$ 136 b$ Abs. 1 Nr. 4 SGB V die vier Leistungsbereiche festgelegt, zu denen Qualitätsverträge zwischen Krankenkassen oder Zusammenschlüsse von Krankenkassen und Krankenhausträgern geschlossen werden dürfen (G-BA 2017). Qualitätsverträge konnten bereits seit Anfang August 2018 (GKV-SV, DKG 2018) geschlossen werden. Wirksam wurden die bis dahin abgeschlossenen Qualitätsverträge jedoch erst im Juli 2019. Der Erprobungszeitraum dauert maximal vier Jahre. Anschließend erfolgt die Evaluation der Ergebnisse durch das Institut für Qualitätssicherung und Transparenz im Gesundheitswesen (IQTIG) oder die lokalen Vertragspartner. 
In den Qualitätsverträgen werden die Qualitätsziele, Anreize und die Auswahl der Qualitätsindikatoren bzw. Kennziffern sowie die Art (monetär/nicht-monetär) und die Struktur der Anreize durch die Vertragspartner festgelegt. Die Qualitätsziele sollten den Qualitätsanforderungen des IQTIG entsprechen und in Anlehnung an die übergreifenden Ziele, definiert durch den G-BA (2017), vereinbart werden. In der Rahmenvereinbarung zwischen GKV-Spitzenverband und DKG heißt es dazu: „In den Qualitätsverträgen sind Anreize zu vereinbaren, die insbesondere die Krankenhausträger motivieren und unterstützen sollen, die definierten Qualitätsanforderungen zu erreichen. Die Ausgestaltung von Anreizen ist als Teil der Qualitätsverträge frei verhandelbar. Es kann sich um nicht-monetäre oder monetäre Anreize handeln. Als mögliche Anreizsysteme kommen z. B. die Empfehlung des Krankenhauses durch die Krankenkasse, einmalige Zahlungen für den Erprobungszeitraum oder Varianten erfolgsabhängiger Zahlungen oder Mischformen in Betracht."

Der erste Qualitätsvertrag wurde im Dezember 2018 zwischen der Karl-Hansen-Klinik in Bad Lippspringe und der Siemens Betriebskrankenkasse (SBK) im Leistungsbereich „Respiratorentwöhnung von langzeitbeatmeten Patientinnen und Patienten“ abgeschlossen (Siemens-Betriebskrankenkasse SBK 2019). Details zu den darin enthaltenen, auf die Qualität gerichteten finanziellen Anreize sind nicht öffentlich zugänglich. Die Ergebnisse von Qualitätsverträgen könnten eine Grundlage für die Entwicklung von „Best Practice“-Tarifen und damit eine Grundlage für die Weiterentwicklung der Vergütung darstellen.

\section{-. Fallzusammenführung \\ bei Wiederaufnahmen}

Außer der neuen Möglichkeit der Qualitätsverträge, die Ergebnisqualität vergütungswirksam zu berücksichtigen, gibt es seit der DRG-Einführung eine andere ergebnisorientierte Vergütungsmodifikation, nämlich die Fallzusammenführung bei ungeplanten Wiederaufnahmen. Keine getrennte Vergütung wird erzielt, wenn insbesondere kurz nach der Entlassung bis zum Erreichen der oberen Grenzverweildauer bzw. innerhalb von 30 Tagen nach Erstaufnahme für eine DRG der medizinischen oder sonstigen Partition eine Wiederaufnahme für eine DRG der gleichen Hauptdiagnosegruppe (MDC) in der operativen Partition stattfindet. In beiden Fällen werden die zwei Aufenthalte $\mathrm{zu}$ nur einem vergütungsfähigen Fall zusammengeführt, im ersten Fall normalerweise unter der erstkodierten DRG, in letzterem unter der letztkodierten DRG. Wie viele Fälle davon in Deutschland betroffen sind (und welchen Anteil an allen Wiederaufnahmen dies betrifft), lässt sich mit öffentlich verfügbaren Daten nicht sagen. Eine Studie mit Daten von 1997 bis 2002, also vor der DRG-Einführung, zeigte, dass $12,7 \%$ aller Patienten innerhalb von 30 Tagen wiederaufgenommen wurden (Nüssler et al. 2006) - das Potenzial ist also sehr groß.

\subsection{Fazit}

Alle Vergütungsformen beinhalten Anreize zur Gefährdung der Qualität. Die Vergütung von Krankenhausfällen - und die genaue Ausgestaltung der ihr innewohnenden Anreize sollte andere qualitätsfördernde Maßnahmen aber unterstützen und nicht konterkarieren. Die bestehenden Vergütungsformen könnten durch Boni, Vergütungsabschläge bis hin zur Nicht-Vergütung, Einbehalte und „shared savings“ modifiziert werden. Während „Pay-forQuality“-Programme in anderen europäischen Ländern hierfür Inspiration und Evidenz nicht nur zur Wirkung, sondern auch zu Nebenwirkungen - liefern, sollte eine grundlegende Orientierung der Krankenhausvergütung an der Qualitätssicherung und -verbesserung aus multiplen Komponenten bestehen, statt auf Programme verengt zu werden, die Krankenhäuser für einzelne Prozess- oder Ergebnisparameter belohnen oder bestrafen. Während das Krankenhausstrukturgesetz die rechtlichen Möglichkeiten hierzu vergrößert hat, ist weiterhin nur ein kleiner Teil der Komponenten umgesetzt. Ein entscheidender Schritt vorwärts 
wäre eine Umstellung sowohl der Krankenhausplanung als auch der -vergütung auf das Prinzip, dass jede Leistung nur von personell und technisch adäquat ausgestatteten Krankenhäusern erbracht werden darf - und vergütet wird. Damit würde eine neu gestaltete Vergütung endlich zum zentralen Motor der notwendigen Krankenhausreform.

\section{Literatur}

Anell A (2013) Vårdval i specialistvården. Utveckling och utmaningar. Sveriges kommuner och landsting, Stockholm. http://webbutik.skl.se/bilder/artiklar/ pdf/7585-007-8.pdf?issuusl=ignore. Zugegriffen: 21. Nov. 2019

AQuA (2017) About us: how does advancing quality measure performance? Advancing quality alliance. http://www.advancingqualitynw.nhs.uk/about-us/. Zugegriffen: 26. Okt. 2019

Armour BS, Pitts MM, Maclean R, Cangialose C, Kishel M, Imai H, Etchason J (2001) The effect of explicit financial incentives on physician behavior. Arch Intern Med 161(10):1261-1266

Barreto JO (2015) Pay-for-performance in health care services. A review of the best evidence available. Ciencia Saude Coletiva 20(5):1497-1514. https://doi.org/10. 1590/1413-81232015205.01652014

Busse R (2016) Pay-for-performance: Time to act but also to provide further evidence (Editorial). Health Policy 120(10):1123-1124

Busse R, Quentin W (2011) Moving towards transparency, efficiency and quality in hospitals: conclusions and recommendations. In: Busse R, Geissler A, Quentin W, Wiley M (Hrsg) Diagnosis-related groups in Europe: moving towards transparency, efficiency and quality in hospitals. Open University Press and WHO Regional Office for Europe, Maidenhead, S 149-171

Christianson JB, Leatherman ST, Sutherland K (2007) Financial incentives, healthcare providers and quality improvements. A review of the evidence. Health Foundation, London. http://www.health.org.uk/ sites/health/files/FinanciallncentivesHealthcare

PRovidersAndQualitylmprovements.pdf. Zugegriffen: 21. Nov. 2019

Christianson JB, Leatherman ST, Sutherland K (2008) Lessons from evaluations of purchaser pay-forperformance programs. a review of the evidence. Med Care Res Rev 65(6 Suppl):5S-35S. https://doi. org/10.1177/1077558708324236

de Cruppé W, Geraedts M (2018) Mindestmengen unterschreiten, Ausnahmetatbestände und ihre Konsequenzen ab 2018. Komplexe Eingriffe am Ösophagus und Pankreas in deutschen Krankenhäusern im Zeit- verlauf von 2006 bis 2014. Zentralbl Chir 143(3):250258. https://doi.org/10.1055/a-0573-2625

de Cruppé W, Malik M, Geraedts M (2015) Minimum volume standards in German hospitals: do they get along with procedure centralization? A retrospective longitudinal data analysis. BMC Health Serv Res 15:279. https://doi.org/10.1186/s12913-015-0944-7

Damberg CL, Sorbero ME, Mehrotra A, Teleki S, Lovejoy SL, Bradley L (2007) An environmental scan of pay for performance in the hospital setting: final report. Hrsg $v$ office of the assistant secretary for planning and evaluation (ASPE). RAND corporation (RAND health working paper series). https://aspe.hhs. gov/report/environmental-scan-pay-performancehospital-setting-final-report. Zugegriffen: 21. Nov. 2019

Damberg CL, Sorbero ME, Lovejoy SL, Martsolf G, Raaen L, Mandel D (2014) Measuring success in health care value-based purchasing programs. Findings from an environmental scan, literature review, and expert panel discussions. RAND Corporation, Washington DC

DeCamp M, Farber NJ, Torke AM, George M, Berger Z, Keirns CC, Kaldjian LC (2014) Ethical challenges for accountable care organizations: a structured review. J GEN INTERN MED 29(10):1392-1399. https://doi. org/10.1007/s11606-014-2833-x

Doran T, Maurer KA, Ryan AM (2017) Impact of provider incentives on quality and value of health care. Annu Rev Public Health 38:449-465. https://doi.org/10. 1146/annurev-publhealth-032315-021457

Eckhardt H, Smith P, Quentin W (2019) Pay for Quality: using financial incentives to improve quality of care. In: Busse R, Klazinga N, Panteli D, Quentin W (Hrsg) Improving healthcare quality in Europe. Characteristics, effectiveness and implementation of different strategies. Health Policy Series 53. World Health Organization and OECD, Copenhagen, S 357-400

Eijkenaar F (2013) Key issues in the design of pay for performance programs. Eur J Health Econ 14(1):117131. https://doi.org/10.1007/s10198-011-0347-6

Ellis RP, Miller MM (2009) Provider Payment Methods and Incentives. In: Carrin G (Hrsg) Health systems poli$c y$, finance, and organization. Elsevier, Amsterdam, S 322-329

Emanuel EJ, Ubel PA, Kessler JB, Meyer G, Muller RW, Navathe AS et al (2016) Using behavioral economics to design physician incentives that deliver high-value care. Ann Intern Med 164(2):114-119. https://doi. org/10.7326/M15-1330

Emmert M, Eijkenaar F, Kemter $H$, Esslinger AS, Schöffski O (2012) Economic evaluation of payfor-performance in health care. A systematic review. Eur J Health Econ 13(6):755-767. https://doi.org/10. 1007/s10198-011-0329-8

FHL (2012) Le modèle des Incitants Qualité - Bilan des démarches communes $\mathrm{EHL}$ - CNS et perspectives. Fédération des Hôpitaux Luxembourgeois (FHL), Lu- 
xembourg. http://www.fhlux.lu/web/wp-content/ uploads/2011/03/SV-Mod\%C3\%A8le-IncitantsQualit\%C3\%A9-CEVAL.pdf. Zugegriffen: 21. Nov. 2019

FOD Volksgezondheid, Veiligheid van de Voedselketen en Leefmilieu (2018) Pay for Performanceprogramma 2018 voor algemene ziekenhuizen. Begeleidende nota. Federale overheidsdienst (FOD) Volksgezondheid, Veiligheid van de Voedselketen en Leefmilieu, Brussel. https://www.health.belgium.be/ sites/default/files/uploads/fields/fpshealth_theme_ file/begeleidende_nota_p4p_24_april_2018.pdf. Zugegriffen: 15. Nov. 2019

G-BA (2017) Tragende Gründe des Gemeinsamen Bundesausschusses über die Festlegung der Leistungen oder Leistungsbereiche gemäß § 136b Abs 1 Satz 1 Nr 4 SGB V für Qualitätsverträge nach § 110a SGB V, vom 18. Mai 2017. https://www.g-ba.de/ beschluesse/2960/. Zugegriffen: 27. Sept. 2019

G-BA (2018) Regelungen des Gemeinsamen Bundesausschusses zu einem gestuften System von Notfallstrukturen in Krankenhäusern gemäß § 136c Abs 4 des Fünften Buches Sozialgesetzbuch (SGB V), vom 19. April 2018. Veröffentlicht im Bundesanzeiger AT 18.05.2018 B4. https://www.g-ba.de/ downloads/62-492-1598/Not-Kra-R_2018-04-19_ iK2018-05-19.pdf. Zugegriffen: 25. Sept. 2019

G-BA (2019) Regelungen des Gemeinsamen Bundesausschusses gemäß § 136b Abs 1 S 1 Nr 2 SGB V für nach § 108 SGB V zugelassene Krankenhäuser (Mindestmengenregelung, Mm-R) vom 20.06.2019. In: Bundesanzeiger (BAnz AT 12.07.2019 B2). https:// www.g-ba.de/downloads/62-492-1882/Mm-R 2019-06-20_iK-2019-07-13.pdf. Zugegriffen: 26. Nov. 2019

Geissler A, Quentin W, Scheller-Kreinsen D, Busse R (2011) Introduction to DRGs in Europe: Common objectives across different hospital systems. In: Busse R, Geissler A, Quentin W, Wiley M (Hrsg) Diagnosis-related groups in Europe. Moving towards transparency, efficiency and quality in hospitals. Open University Press (European Observatory on Health Systems and Policies series), Maidenhead, S 9-21

Giuffrida A, Gosden T, Forland F, Kristiansen IS, Sergison M, Leese B et al (2000) Target payments in primary care. Effects on professional practice and health care outcomes. Cochrane Database Syst Rev. https://doi. org/10.1002/14651858.cd000531

GKV-SV, DKG (2018) Vereinbarung über die verbindlichen Rahmenvorgaben nach § 110a Abs 2 SGB V für den Inhalt der Qualitätsverträge nach § 110a Abs 1 SGB V. Rahmenvereinbarung für Qualitätsverträge in der stationären Versorgung vom 16.07.2018. https:// www.gkv-spitzenverband.de/media/dokumente/ krankenversicherung_1/krankenhaeuser/ qualitaetsvertraege/Rahmenvereinbarung_ fuer_Qualitaetsvertraege_in_der_stationaeren_
Versorgung_16.07.2018.pdf. Zugegriffen: 27. Sept. 2019

Hamilton FL, Greaves F, Majeed A, Millett C (2013) Effectiveness of providing financial incentives to healthcare professionals for smoking cessation activities. Systematic review. Tob Control 22(1):3-8. https://doi. org/10.1136/tobaccocontrol-2011-050048

Hertle A, Veit C (2012) Pay-for-Performance in Deutschland: Von der Option zur Umsetzung. Monit Versorgungsforsch 5(6):40-46

Houle SK, McAliste FA, Jackevicius CA, Chuck AW, Tsuyuki RT (2012) Does performance-based remuneration for individual health care practitioners affect patient care? A systematic review. Ann Intern Med 157(12):889-899. https://doi.org/10.7326/ 0003-4819-157-12-201212180-00009

IGES (2018) Folgenabschätzung einer gestuften Notfallversorgung. Folgenabschätzung für die Regelungen für ein gestuftes System von Notfallstrukturen in Krankenhäusern gemäß § 136c Abs 4 SGB V. Unter Mitarbeit von Dr. Martin Albrecht. IGES Institut GmbH, Berlin. https://www.g-ba.de/beschluesse/ 3401/letzte-aenderungen/. Zugegriffen: 23. Sept. 2019

Joynt Maddox KE, Sen AP, Samson LW, Zuckerman RB, DeLew N, Epstein AM (2017) Elements of program design in medicare's value-based and alternative payment models: a narrative review. J Gen Int Med 32(11):1249-1254. https://doi.org/10.1007/s11606017-4125-8

Kohn LT, Corrigan J, Donaldson MS (2000) To err is human. Building a safer health system. Quality chasm series. National Academy Press, Washington, DC

Kondo K, Damberg C, Mendelson A, Motu'apuaka M, Freeman M, O'Neil M et al (2015) Understanding the intervention and implementation factors associated with benefits and harms of pay for performance programs in Healthcare. Department of Veterans Affairs, US

Korenstein D, Duan K, Diaz MJ, Ahn R, Keyhani S (2016) Do health care delivery system reforms improve value? The jury is still out. Med Care 54(1):55-66. https://doi. org/10.1097/mlr.0000000000000445

Krauth C, Schwartz FW, Perleth M, Busse R, von der Schulenburg J-M (1998) Zur Umsetzung ergebnisorientierter Vergütungselemente in der ambulanten Versorgung. Arb Sozialpolitik 52(11/12):10-22

Kristensen SR, Bech M, Lauridsen JT (2016) Who to pay for performance? The choice of organisational level for hospital performance incentives. Eur J Health Econ 17(4):435-442. https://doi.org/10.1007/s10198-0150690-0

Langdown C, Peckham S (2014) The use of financial incentives to help improve health outcomes: is the quality and outcomes framework fit for purpose? A systematic review. J Public Health 36(2):251-258. https://doi.org/10.1093/pubmed/fdt077 
MDK Nordrhein (2018) Jahresbericht 2018. Düsseldorf. https://www.mdk-nord-rhein.de/ fileadmin/redaktion/Presse/Publikationen/NRH_ 24_JAHRESBERICHT_2018_12_WEB.pdf. Zugegriffen: 13. Nov. 2019

Mechanic D, Schlesinger M (1996) The impact of managed care on patients' trust in medical care and their physicians. JAMA 275(21):1693-1697. https:// doi.org/10.1001/jama.1996.03530450083048

Mehrotra A, Damberg CL, Sorbero ME, Teleki SS (2009) Pay for performance in the hospital setting: what is the state of the evidence? Am J Med Qual 24(1):1928. https://doi.org/10.1177/1062860608326634

Milstein R, Schreyögg J (2016) Pay for performance in the inpatient sector: a review of $34 \mathrm{P} 4 \mathrm{P}$ programs in 14 OECD countries. Health Policy 120(10):1125-1140. https://doi.org/10.1016/j.healthpol.2016.08.009

Ministère des Affaires Sociales et de la Santé (2016) Arrêté du 5 août 2016 fixant les modalités de calcul du montant de la dotation allouée aux établissements de santé en application de l'article L. 162-2220. Journal Officiel de la République Française (0192). https://www.legifrance.gouv.fr/affichTexte. do?cidTexte=JORFTEXT000033047506. Zugegriffen: 21. Nov. 2019

MSPY (2016) National Social Report 2016 Republic of Croatia. Ministry of Social Policy and Youth, Zagreb. https://www.google.de/url?sa=t\&rct=j\&q=\& esrc $=s \&$ source $=$ web $\& c d=3 \& c a d=r j a \& u a c t=8 \&$ ved $=$ OahUKEwitnf765IvYAhVRpKQKHQc2DgwQFgg3MAI\& url=http\%3A\%2F\%2Fec.europa.eu\%2F social\%2FBlobServlet\%3Fdocld\%3D16483\%26 langld\%3Den\&usg=AOvVaw1qRQ3nbUIOdc4hTPvD4c-. Zugegriffen: 21. Nov. 2019

England NHS, NHS Improvement (2019) 2019/20 national tariff payment system - a consultation notice: annex DtD guidance on best practice tariffs. A joint publication by NHS england and NHS improvement. https:// improvement.nhs.uk/documents/484/Annex_DtD_ Best_practice_tariffs.pdf. Zugegriffen: 21. Nov. 2019

NHS Improvement (2019) 2019/20 National Tariff Payment System. https://improvement.nhs.uk/ resources/national-tariff/. Zugegriffen: 11. Nov. 2019

Nimptsch U, Peschke D, Mansky T (2017) Mindestmengen und Krankenhaussterblichkeit - Beobachtungsstudie mit deutschlandweiten Krankenhausabrechnungsdaten von 2006 bis 2013. Gesundheitswesen 79(10):823-834. https://doi.org/10.1055/s-0042100731

Nüssler NC et al (2006) Mehr Wiederaufnahmen nach Entlassung am Freitag. Dt Ärztebl 103(14):A927-A931
OECD (2018) Health at a glance. Europe 2018: state of health in the EU cycle. OECD Publishing, Paris

Ogundeji YK, Bland JM, Sheldon TA (2016) The effectiveness of payment for performance in health care: A meta-analysis and exploration of variation in outcomes. A meta-analysis and exploration of variation in outcomes. Health Policy 120(10):1141-1150. https://doi.org/10.1016/j.healthpol.2016.09.002

Olsen CB, Brandborg G (2016) Quality based financing in Norway. Country background note: Norway. Norwegian directorate of health. https://www.oecd.org/ els/health-systems/Better-Ways-to-Pay-for-HealthCare-Background-Note-Norway.pdf. Zugegriffen: 21. Nov. 2019

Petersen LA, Woodard LD, Urech T, Daw C, Sookanan S (2006) Does pay-for-performance improve the quality of health care? Ann Intern Med 145(4):265-272

Roland M, Dudley RA (2015) How financial and reputational incentives can be used to improve medical care. Health Serv Res 50(Suppl 2):2090-2115. https://doi. org/10.1111/1475-6773.12419

Rosenthal MB, Frank RG, Li Z, Epstein AM (2005) Early experience with pay-for-performance: from concept to practice. JAMA 294(14):1788-1793

Rosenthal MB, Landon BE, Howitt K, Ryu SH, Epstein AM (2007) Climbing up the pay-for-performance learning curve: where are the early adopters now? Health Aff 26(6):1674-1682

Schreyögg J, Busse R, Bäuml M, Krämer J, Dette T, Geissler A (2014) Forschungsauftrag zur Mengenentwicklung nach § 17b Abs. 9 KHG. Endbericht. Hamburg Center for Health Economics; Technische Universität Berlin. https://www.g-drg.de/Datenbrowser_ und_Begleitforschung/Begleitforschung_DRG/ Forschungsauftrag_gem._17b_Abs._9_KHG. Zugegriffen: 2. Okt. 2019

Siemens-Betriebskrankenkasse SBK (2019) Erster Qualitätsvertrag zur Beatmungsentwöhnung geöffnet. Pressemitteilung: Ab sofort können weitere Kliniken und Krankenkassen dem Vertrag der SBK beitreten. https://www.sbk.org/unserethemen/versorgung/erster-qualitaetsvertrag-zurbeatmungsentwoehnung-geoeffnet. Zugegriffen: 27. Sept. 2019

Srivastava D, Mueller M, Hewlett E (2016) Better ways to pay for health care. OECD Publishing, Paris

van Herck P, de Smedt D, Annemans L, Remmen R, Rosenthal MB, Sermeus W (2010) Systematic review: effects, design choices, and context of pay-forperformance in health care. BMC Health Serv Res 10:247. https://doi.org/10.1186/1472-6963-10-247 
Open Access Dieses Kapitel wird unter der Creative Commons Namensnennung 4.0 International Lizenz (http:// creativecommons.org/licenses/by/4.0/deed.de) veröffentlicht, welche die Nutzung, Vervielfältigung, Bearbeitung, Verbreitung und Wiedergabe in jeglichem Medium und Format erlaubt, sofern Sie den/die ursprünglichen Autor(en) und die Quelle ordnungsgemäß nennen, einen Link zur Creative Commons Lizenz beifügen und angeben, ob Änderungen vorgenommen wurden.

Die in diesem Kapitel enthaltenen Bilder und sonstiges Drittmaterial unterliegen ebenfalls der genannten Creative Commons Lizenz, sofern sich aus der Abbildungslegende nichts anderes ergibt. Sofern das betreffende Material nicht unter der genannten Creative Commons Lizenz steht und die betreffende Handlung nicht nach gesetzlichen Vorschriften erlaubt ist, ist für die oben aufgeführten Weiterverwendungen des Materials die Einwilligung des jeweiligen Rechteinhabers einzuholen. 